\title{
VII. Die Professionalisierung des Militärs 1961-1965
}

\section{Die Integration der NVA in die Erste Strategische Staffel des Warschauer Paktes}

Die Integration der NVA in die Erste Strategische Staffel der Vereinten Streitkräfte des Warschauer Paktes ${ }^{1}$ war für die weitere Entwicklung der zivil-militärischen Beziehungen in der DDR ab 1961 von entscheidender Bedeutung. Durch sie rückte die Verbesserung der Einsatzbereitschaft der NVA ins Zentrum der Militärpolitik der SED.

In der Praxis scheint sich die Integration nicht als einfache Zuordnung von Verbänden und Truppenteilen der NVA zu den Einheiten der Ersten Strategischen Staffel vollzogen zu haben. Vielmehr erfolgte sie offenbar als ein „Prozeß der Eingliederung", der 1961 eingeleitet wurde². Von diesem Zeitpunkt an gehörte die NVA formal zur vordersten, nach Westen gerichteten Angriffsformation des Warschauer Paktes in Mitteleuropa ${ }^{3}$, ohne aber eigenständig operieren zu können. Die Landstreitkräfte der NVA bildeten dabei zusammen mit der GSSD die 1. Front $t^{4} \mathrm{der} \mathrm{Er}-$ sten Strategischen Staffel auf dem Territorium der DDR. Einige NVA-Einheiten waren außerdem der 2. Front (Küstenfront) zugeordnet, die vor allem die Polnische Armee stellte. Zudem wurde die Volksmarine Bestandteil der Vereinten Ostseeflotten unter dem Kommando des Chefs der Baltischen Flotte der UdSSR. Operativ war sie damit ebenfalls dem Befehlshaber der 1. Front unterstellt. Die Luftstreitkräfte übernahmen ab 1962 aktiv Aufgaben im einheitlichen System der Luftverteidigung der Warschauer Pakt-Staaten ${ }^{5}$. Die Integration der NVA in die Erste Strategische Staffel wurde dann offenbar innerhalb von fünf Jahren weitgehend abgeschlossen. Ihr Ende markierte das Manöver „Oktobersturm“ im Oktober 1965.

Unklar bleibt, ob der Integrationsprozeß bereits vor dem Mauerbau am 13. August 1961 in Gang gesetzt wurde. Für diese Annahme sprechen die militärischen Maßnahmen in der ersten Jahreshälfte. So hatte der Nationale Verteidigungsrat schon am 20. Januar 1961 entsprechend einer Empfehlung des Vereinten Kommandos „Änderungen in der Struktur und in der Bewaffnung und Ausrüstung“ der

1 Vgl. u. a. Wenzke, Die Nationale Volksarmee, S. 448 ff., 483 f.; Arlt, Sowjetische (russische) Truppen in Deutschland (1945-1994), S. $608 \mathrm{f} ., 614 \mathrm{f}$.

2 Greese/Voerster, Probleme der Auswahl und Förderung der Offizierskader, S. 44.

3 Teilbeitrag der Verwaltung Kader zur Einarbeitung in die Gesamtanalyse und Grundkonzeption der Entwicklung der NVA, 10. März 1967, BA-MA, VA-01/5679, Bl. 9. Insofern ist der Auffassung von Peter Joachim Lapp zu widersprechen, die Landstreitkräfte der NVA hätten seit 1965 zur Ersten Strategischen Staffel des Warschauer Paktes gehört. Lapp, Die Nationale Volksarmee 1956-1990, S. 1906.

4 Die Front ist vergleichbar mit einer Heeresgruppe der NATO.

5 Wenzke, Die Nationale Volksarmee, S. 483. 
NVA beschlossen ${ }^{6}$. Vermutlich ging es darum, im Hinblick auf die Einbeziehung in die Fronten der Ersten Strategischen Staffel eine bessere Kompatibilität der NVATruppenteile mit den Einheiten der sowjetischen Streitkräfte herzustellen. Der Beschluß dürfte durch die Aufforderung des Politischen Beratenden Ausschusses an die Mitgliedsstaaten des Warschauer Paktes Ende März 1961, ihre Streitkräfte weiter zu modernisieren, einen zusätzlichen Schub erfahren haben ${ }^{7}$.

Darüber hinaus fand vom 23. bis 30. Mai 1961 eine gemeinsame Kommandostabs- und Truppenübung der GSSD und der NVA statt. Bei dieser Übung könnte es sich um den praktischen Beginn der Integration der NVA in die Erste Strategische Staffel gehandelt haben. In diesem Sinne äußerte Ulbricht hinsichtlich der Übung: „Wir schätzen sie als das wichtigste Ereignis im Ausbildungsjahr ein. Mit ihr wurde eine neue Etappe in der Entwicklung der Nationalen Volksarmee und im Zusammenwirken mit der Gruppe der sowjetischen Streitkräfte in Deutschland eingeleitet."8

Vor allem dürfte es bei dieser Übung darum gegangen sein, eine Bestandsaufnahme des aktuellen Standes der Einsatzbereitschaft und der operativen Fähigkeiten der NVA vorzunehmen, um den Eingliederungsprozeß gezielt weiter vorantreiben zu können. So basierte die Übung auf dem Ausbildungsprogramm des Stabes der Vereinten Streitkräfte. In der Ausgangslage ging man von einer realistischen Dislozierung der Streitkräfte aus. Die Übungsanlage sah die Abwehr eines überraschenden Angriffs der NATO vor. Angenommen wurden dabei unter anderem über 900 Atomschläge beider Seiten. Die Übung stand unter der Leitung des Oberkommandierenden der Vereinten Streitkräfte, Gretschko, wobei der Minister für Nationale Verteidigung der DDR, Hoffmann, als sein Stellvertreter eingesetzt war?.

Ferner läßt sich die Bedeutung, die die Übung im Rahmen der Integration hatte, auch daran ablesen, daß die NVA und die sowjetischen Streitkräfte unter anderem zwei komplette Divisionen aufboten ${ }^{10}$. Die NVA setzte insgesamt 13000 Soldaten, rund 350 Panzer und über $2800 \mathrm{Kraftfahrzeuge} \mathrm{ein.} \mathrm{Laut} \mathrm{Hoffmann} \mathrm{war} \mathrm{ein} \mathrm{Haupt-}$ ziel des Manövers im Hinblick auf die NVA die „Überprüfung des Standes der Kenntnisse und Fähigkeiten in der Truppenführung" der Stäbe in der Anfangsperiode eines Krieges. Anscheinend standen hierbei das Ministerium für Nationale Verteidigung und insbesondere sein Hauptstab im Mittelpunkt des Interesses. Zudem sollte der Ausbildungsstand des Kommandos der Luftstreitkräfte/Luftverteidigung sowie des Kommandos der Volksmarine kontrolliert werden ${ }^{11}$.

6 Protokoll der 4. Sitzung des Nationalen Verteidigungsrates der DDR, 20. Januar 1961, BA-MA, DVW 1/39461, Bl. 4.

7 Armee für Frieden und Sozialismus, S. 294.

8 Auswertung der gemeinsamen Kommandostabsübung vom 23. bis 30. Mai 1961, 1961, BA-MA, VA-01/6301, Bl. 27.

9 Bericht des Ministers für Nationale Verteidigung an den Nationalen Verteidigungsrat der DDR über die gemeinsame zweistufige Kommandostabsübung vom 23. bis 30. Mai 1961, 1961, BA-MA, VA-01/6301, Bl. 2, 4.

10 Bericht über die gemeinsame zweiseitige Truppenübung in der Zeit vom 25. bis 27. Mai 1961. Protokoll der Sitzung des Kollegiums des Ministeriums für Nationale Verteidigung, 12. Juni 1961, BA-MA, AZN/28185, Bl. 27.

11 Bericht des Ministers für Nationale Verteidigung an den Nationalen Verteidigungsrat der DDR über die gemeinsame zweistufige Kommandostabsübung vom 23. bis 30. Mai 1961, 1961, BA-MA, VA-01/6301, Bl. 3 f. 
Die Übung zeigte, daß die NVA zu diesem Zeitpunkt die Voraussetzungen erfüllte, um "gemeinsame Handlungen mit den sowjetischen Truppen durchzuführen und das Zusammenwirken mit ihnen sicherzustellen" 12 . Als Fazit hieß es, die NVA sei ,im allgemeinen in der Lage, unter den Bedingungen eines modernen Krieges zu handeln"13. Gretschko schätzte die Kenntnisse der NVA-Offiziere mit „befriedigend" ein ${ }^{14}$. Moniert wurde aber andererseits, die Truppenführung sei nicht in jeder Lage aufrecht erhalten worden. Zudem habe das Forcieren der Elbe „nicht voll den Bedingungen eines modernen Krieges" entsprochen ${ }^{15}$ und die Gefechtsordnung eine „noch zu lineare Form“ aufgewiesen ${ }^{16}$. Auch habe die Übung gezeigt, daß „eine planmäßige Mobilmachung noch nicht möglich gewesen wäre"17.

Nach dem Mauerbau wurde der Integrationsprozeß mit Nachdruck weiter vorangetrieben. Der Nationale Verteidigungsrat beschloß am 28. August 1961, die vom Oberkommandierenden der Vereinten Streitkräfte gestellten Aufgaben „zum kürzesten Termin“ zu erfüllen. Dazu gehörte vor allem die „Erhöhung der Gefechtsbereitschaft" aller Teilstreitkräfte, die Vorbereitung zur Aufstellung zusätzlich erforderlicher Truppenteile und Einheiten sowie die Ausarbeitung eines „operativen Planes“ der NVA ${ }^{18}$. Im Februar 1963 wurden schließlich anläßlich einer Beratung des Vereinten Kommandos mit Vertretern des Ministeriums für Nationale Verteidigung die Rahmenbedingungen für die weitere Entwicklung der DDR-Streitkräfte fixiert: Diese sollten eine Friedensstärke von 105000 Mann besitzen ${ }^{19}$, wobei die seit 15. September 1961 zur NVA gehörenden Grenztruppen ${ }^{20}$ im Frieden nicht zum Bestand der Vereinten Streitkräfte zählten. Die Kriegsstärke der NVA wiederum betrug 200000 Mann. Im Kriegsfall zählten „alle bestehenden und neu aufzustellen-

12 Ebenda, Bl. 14.

13 Bericht über die gemeinsame zweiseitige Truppenübung in der Zeit vom 25. bis 27. Mai 1961. Protokoll der Sitzung des Kollegiums des Ministeriums für Nationale Verteidigung, 12. Juni 1961, BA-MA, AZN/28185, Bl. 32.

14 Bericht des Ministers für Nationale Verteidigung an den Nationalen Verteidigungsrat der DDR über die gemeinsame zweistufige Kommandostabsübung vom 23. bis 30. Mai 1961, 1961, BA-MA, VA-01/6301, Bl. 18.

15 Ebenda, Bl. 14.

16 Bericht über die gemeinsame zweiseitige Truppenübung in der Zeit vom 25. bis 27. Mai 1961. Protokoll der Sitzung des Kollegiums des Ministeriums für Nationale Verteidigung, 12. Juni 1961, BA-MA, AZN/28185, Bl. 32.

17 Auswertung der gemeinsamen Kommandostabsübung vom 23. bis 30. Mai 1961. Protokoll der Sitzung des Kollegiums des Ministeriums für Nationale Verteidigung, 12. Juni 1961, BA-MA, AZN/28185, Bl. 17. Ende Mai 1961 nahmen darüber hinaus auch Stäbe und Einheiten der Luftstreitkräfte und Luftverteidigung der NVA zum ersten Mal an einer gemeinsamen Luftverteidigungsübung mit Truppen der GSSD teil, die unter der Leitung des Oberkommandierenden der Truppen der Luftverteidigung des Warschauer Paktes stand. Zeittafel zur Militärgeschichte, S. 138.

18 Beschluß Nr. 42/61 des Nationalen Verteidigungsrates der DDR. Protokoll der 7. Sitzung des Nationalen Verteidigungsrates der DDR, 28. August 1961, BA-MA, DVW 1/39464, Bl. 10.

19 Protokoll der Beratung mit der Delegation des Ministeriums für Nationale Verteidigung der DDR über Fragen der weiteren Entwicklung der Streitkräfte der DDR, die sich aus dem Warschauer Vertrag ergeben, 27. Februar 1963, BA-MA, AZN/32592, Bl. 8.

20 Protokoll über die Übergabe der Deutschen Grenzpolizei durch das Ministerium des Innern und die Übernahme der Grenztruppen durch das Ministerium für Nationale Verteidigung. Protokoll der 8. Sitzung des Nationalen Verteidigungsrates der DDR, 29. November 1961, BA-MA, DVW 1/39465, Bl. 100. 
den Verbände und Truppenteile der Streitkräfte der Deutschen Demokratischen Republik" zum Bestand der Vereinten Streitkräfte. Das Protokoll über die gemeinsame Beratung sah ferner vor, daß die Ausstattung der NVA mit moderner Bewaffnung und die Motorisierung der Armee in offenbarer Übereinstimmung mit dem Integrationszeitplan „im wesentlichen“ bis 1965 abgeschlossen sein sollte21.

Von 1961/1962 an nahm die NVA auch an größeren Übungen und Manövern der Vereinten Streitkräfte teil, in die neben den sowjetischen Streitkräften auch die Polnische Armee und die Tschechoslowakische Volksarmee einbezogen waren. Die erste dieser Übungen der vier Warschauer Pakt-Staaten, die gemeinsame Kommandostabsübung „Burja“, fand unmittelbar nach dem Mauerbau vom 28. September bis 10. Oktober 1961 statt. Ihr Hauptziel war die Überprüfung der operativen Fähigkeiten der Stäbe zur Führung integrierter Truppenverbände unter komplizierten Lagebedingungen ${ }^{22}$. Das zweite wichtige Manöver war die Übung -Vitr“ der Streitkräfte der CSSR (Tschechoslowakische Sozialistische Republik), der DDR und der UdSSR vom 23. bis 27. September 1962. Im Rahmen dieser Übung, bei der insgesamt 35000 Soldaten eingesetzt wurden, handelten erstmals Einheiten der drei Armeen unter einer einheitlichen Truppenführung im operativen Rahmen ${ }^{23}$. Dabei übten sie die „Organisation der Angriffsoperationen einer allgemeinen Armee in der Anfangsperiode eines Krieges" und das Führen von Begegnungsschlachten. Im Ergebnis des Manövers stellte man von Seiten der NVA fest: „Die Zusammenarbeit mit der Volksarmee der ČSSR konnte noch nicht in vollem Umfang befriedigen." 24

Von Bedeutung für die weitere Integration der NVA in die Erste Strategische Staffel war zudem die Übung „Baltyk-Odra“ vom 5. bis 10. Oktober 1962, an der die Flotten der DDR, der UdSSR und Polens teilnahmen. Nach Einführung gemeinsamer Einsatzgrundsätze für die drei Seestreitkräfte des Warschauer Paktes in der Ostsee wurde bei diesem Manöver erstmals deren Zusammenwirken mit den übrigen Teilstreitkräften geübt ${ }^{25}$. Thema der Übung war die gemeinsame Abwehr einer großen gegnerischen "Seelandungs- und Luftlandeoperation“ an der polnischen Küste auf Armeeebene und das Übergehen eines "gemischten operativen Verbandes" zum Gegenangriff entlang der Küstenlinie während der Anfangsphase eines Krieges 26 .

Im Frühjahr 1962 wurden Teile der beiden neuformierten Luftverteidigungsdivisionen der NVA in das „Diensthabende System der Luftverteidigung“ des Warschauer Paktes eingegliedert. Die Luftstreitkräfte/Luftverteidigung der NVA hatten von nun an die Aufgabe, zusammen mit der GSSD rund um die Uhr den DDR-

21 Protokoll der Beratung mit der Delegation des Ministeriums für Nationale Verteidigung der DDR über Fragen der weiteren Entwicklung der Streitkräfte der DDR, die sich aus dem Warschauer Vertrag ergeben, 27. Februar 1963, BA-MA, AZN/32592, Bl. $8 \mathrm{f}$.

22 Zeittafel zur Militärgeschichte, S. 147.

23 Ebenda, S. 162.

24 Bericht über die durchgeführte Kommandostabsübung der 7. PD mit Truppen unter Leitung der Volksarmee der ČSSR, 2. Oktober 1962, BA-MA, VA-01/5193, Bl. 114.

25 Vego, Flottenübungen des Warschauer Paktes seit 1956, S. 311.

26 Auswertung der zweiseitigen Armeeübung mit Truppen der vereinigten Streitkräfte der Armeen des Warschauer Vertrages „Baltyk-Odra" vom 5. bis 10. Oktober 1962 durch den Chef des Generalstabes der Polnischen Volksarmee, Waffengeneral J. Bordzilowski, 10. Oktober 1962, BA-MA, VA-01/5194, B1. 151. 
Luftraum zu sichern ${ }^{27}$. In Struktur und Ausrüstung stimmten die Luftverteidigungskräfte der NVA jetzt weitgehend mit denen der übrigen Armeen des Warschauer Paktes überein ${ }^{28}$.

Offenbar war das 1957 geschaffene einheitliche System der Luftverteidigung des Warschauer Paktes bis zu diesem Zeitpunkt den Erwartungen hinsichtlich seiner Leistungsfähigkeit nicht gerecht geworden. Dies mag zudem als ein Indiz dafür gelten, daß es dem Bündnis im Vergleich zur NATO schwerer fiel, integrierte Militärstrukturen zu schaffen. Als Grund für die Probleme in diesem Fall nannte Gretschko Ende 1961, man habe statt einer einheitlichen Leitung seinerzeit „lediglich die Koordinierung von Fragen der Luftverteidigung im System des Kommandos der Vereinten Streitkräfte" vereinbart. Die Übungen der Jahre 1960/1961 hätten dann jedoch gezeigt, daß die Luftverteidigung des Bündnisses „faktisch kein einheitlich abgestimmtes System“ sei und „jedes Land die Aufgaben selbständig und isoliert" löse. Er erachtete es daher als notwendig, in Zukunft die Luftverteidigungstruppen des Warschauer Vertrages auf der Grundlage eines „einheitlichen operativen Planes" in einem abgeschlossenen System zusammenzufassen ${ }^{29}$. Die Verwirklichung dieses Reformvorschlages wurde im Januar 1962 auf der Tagung der Verteidigungsminister der Warschauer Pakt-Staaten beschlossen ${ }^{30}$. Bei der Implementierung scheinen sich dann aber Schwierigkeiten ergeben zu haben, zumindest traten die Veränderungen erst am 1. Mai des darauffolgenden Jahres in $\mathrm{Kraft}^{31}$.

Zum einheitlichen System der Luftverteidigung gehörten nunmehr „alle Truppen der Luftverteidigung" der Staaten des Warschauer Paktes. Im Hinblick auf die sowjetischen Streitkräfte gab es jedoch eine substantielle Einschränkung. Hier wurden nur die in den westlichen Republiken der UdSSR stationierten Einheiten in das System einbezogen. Neben der Sicherung des eigenen Territoriums hatten die Luftverteidigungstruppen jedes Teilnehmerstaates "mit einem Teil der Kräfte“ auch die Luftverteidigung der Nachbarstaaten zu verstärken. Ihr Einsatz sowohl bei der Luftraumüberwachung als auch im Gefecht sollte "nach einem einheitlichen operativen Plan" organisiert werden. Dabei unterlagen die gemeinsamen Gefechtshandlungen einer „zentralisierten Führung“. In der „Bestimmung über das einheitliche System der Luftverteidigung der Teilnehmerstaaten des Warschauer Vertrages“ wurde jedoch ausdrücklich darauf hingewiesen, daß „die Prinzipien der Unterstellung der Truppen der Luftverteidigung eines jeden Staates unter die nationale Führung" nicht zu verletzen seien. Auch behielten die nationalen Armeeführungen die „volle Verantwortung" für die Sicherung des Staatsgebietes sowie für den Zustand der eigenen Luftverteidigungstruppen „sowohl in Friedens- als auch zu Kriegszeiten ${ }^{* 32}$.

$27 \mathrm{Vgl}$. Protokoll der 2. Tagung der IV. Delegiertenkonferenz der Parteiorganisationen der SED in der NVA, 1./2. Dezember 1962, BA-MA, VA-P-01/559, Bl. 107.

28 Wenzke, Die Nationale Volksarmee, S. 446f.

29 Gretschko an Hoffmann, 22. Dezember 1961, BA-MA, VA-01/8758, Bl. 78 f.

30 Beschluß der Tagung der Verteidigungsminister des Warschauer Vertrages, die in Prag vom 30. bis 31. Januar 1962 stattfand, BA-MA, VA-01/8758, Bl. $55 \mathrm{f}$.

31 Mitteilung des Oberkommandos der Vereinten Streitkräfte der Teilnehmerstaaten des Warschauer Vertrages an Hoffmann, 16. Oktober 1963, BA-MA, AZN/32597, Bl. 28.

32 Bestimmung über das einheitliche System der Luftverteidigung der Teilnehmerstaaten des Warschauer Vertrages. Brief des Ministers für Nationale Verteidigung des Polnischen Volks- 
Die Leitung des einheitlichen Systems der Luftverteidigung des Warschauer Paktes unterstand jedoch dem „Oberkommandierenden der Truppen der Luftverteidigung der Teilnehmerstaaten des Warschauer Vertrages“, der zugleich ein Stellvertreter des Oberkommandierenden der Vereinten Streitkräfte war ${ }^{33}$. Aufgrund der Tatsache, daß die Regierungen vereinbart hatten, daß in diese Dienststellung der „Oberkommandierende der Truppen der Luftverteidigung der Sowjetunion“ einzusetzen war $^{34}$, dessen Stab zugleich als Führungsorgan der integrierten Luftverteidigung fungierte ${ }^{35}$, standen das einheitliche Luftverteidigungssystem und damit ebenso die nationalen Luftverteidigungskräfte - so auch die der DDR - unter der direkten Kontrolle der sowjetischen Streitkräfte.

Eine besondere Zäsur bei der Integration der NVA in die Erste Strategische Staffel stellte das Manöver "Quartett" dar, das vom 9. bis 14. September 1963 im Süden der DDR stattfand. Nach Angaben des Chefs des Hauptstabes, Generalleutnant Siegfried Riedel, war es das „bedeutendste Ereignis in der bisherigen operativ-taktischen Ausbildung der NVA"36. An der bis dahin größten Truppenübung der Vereinten Streitkräfte in der DDR nahmen neben der NVA die sowjetischen, polnischen und tschechoslowakischen Streitkräfte mit insgesamt rund 41000 Soldaten, 700 Panzern, 8300 Schützenpanzern und Kraftfahrzeugen sowie 323 Flugzeugen und Hubschraubern teil. Erstmals wurden Truppen der Land- und Luftstreitkräfte sowie der Luftverteidigung der vier Armeen gemeinsam eingesetz ${ }^{37}$.

Geübt wurde einmal mehr die Abwehr eines unter Einsatz von Atomwaffen überraschend vorgetragenen Überfalls der NATO auf das Territorium der DDR. Die Kräfte der Vereinten Streitkräfte sollten nach Märschen über große Entfernungen eine aktive Verteidigung aufbauen und zum direkten Gegenangriff übergehen. Dabei hatten sie, unterstützt von Luftlandetruppen und massiven Luftschlägen, aus der Bewegung Wasserhindernisse auf breiter Front zu überwinden und die Verteidigungsstellungen des Gegners zu durchbrechen. Dies war ein Übungsschwerpunkt, der sich offensichtlich an den geographischen Bedingungen der innerdeutschen Grenze orientierte. Das Manöver endete mit einem Begegnungsgefecht, in dem die gegnerischen Streitkräfte mit hoher Geschwindigkeit aufeinandertrafen ${ }^{38}$. Ziel der Übung war es dabei, die gemeinsamen Einsatzgrundsätze weiter zu harmonisieren und das Zusammenwirken im Bündnisrahmen zu verbessern. Vor allem sollte entsprechend den Weisungen des Oberkommandierenden der Vereinten Streitkräfte

republik Armeegeneral Marian Spychalski an Hoffmann, 19. September 1963, BA-MA, AZN/32592, Bl. $73 \mathrm{ff}$.

33 Ebenda.

34 Protokoll über die Schaffung des einheitlichen Systems der Luftverteidigung der Teilnehmerstaaten des Warschauer Vertrages. Brief des Ministers für Nationale Verteidigung der Polnischen Volksrepublik Armeegeneral Marian Spychalski an Hoffmann, 19. September 1963, BA-MA, AZN/32592, B1. 70.

35 Bestimmung über das einheitliche System der Luftverteidigung der Teilnehmerstaaten des Warschauer Vertrages. Brief des Ministers für Nationale Verteidigung des Polnischen Volksrepublik Armeegeneral Marian Spychalski an Hoffmann, 19. September 1963, BA-MA, AZN/32592, Bl. 75.

36 Bericht über die in der Zeit vom 11. bis 15. September 1963 durchgeführte Übung „Quartett", 18. Januar 1964, BA-MA, VA-01/12946, Bl. 97.

37 Haack, Zur Rolle sowjetischer Erfahrungen, S. 18.

38 Armee für Frieden und Sozialismus, S. $338 \mathrm{ff}$. 
überprüft werden, inwieweit die Stäbe und Truppen der vier Armeen mit ihrer einheitlichen Ausrüstung und Ausbildung dazu in der Lage waren, "gemeinsame Kampfaufgaben zu erfüllen" 39 .

Eine entscheidende Intention des Manövers bestand im übrigen darin, Aufschluß über den aktuellen Stand der Einsatzbereitschaft der NVA im Bündnisrahmen und die operativen Fähigkeiten ihrer Führung zu erhalten. Daher waren neben Gretschko als Oberkommandierendem der Vereinten Streitkräfte Generale und Offiziere der übrigen Warschauer Pakt-Armeen als Beobachter anwesend. Das Vereinte Kommando hatte zum ersten Mal den Minister für Nationale Verteidigung der DDR mit der Leitung einer derartigen Übung beauftragt. Zudem unterstanden die teilnehmenden Kräfte der Luftstreitkräfte Generalleutnant Heinz Keßler, der 1957-1967 den Posten des Chefs der Luftstreitkräfte/Luftverteidigung der NVA bekleidete $^{40}$. Im Gegensatz zu den vorhergehenden Übungen waren jetzt erstmals auch sowjetische Einheiten kommandierenden Generalen der NVA direkt unterstellt. Hierbei handelte es sich um die Chefs der Militärbezirke III und V, die als Befehlshaber von Armeen eingesetzt waren ${ }^{41}$.

In der Auswertung der Übung gelangte Gretschko zu dem Ergebnis, die NVA habe ihre Aufgaben vorbildlich erfüllt und unterscheide sich nicht mehr von den anderen Armeen des Bündnisses ${ }^{42}$. Hinsichtlich der Einschätzung des Leistungsstandes der NVA heißt es im Bericht des Ministeriums für Nationale Verteidigung, das Manöver habe bewiesen, „daß sich die Oberbefehlshaber, Kommandeure und Stäbe der 3. und 5. Armee (MB III und V) und der in ihrem Bestand handelnden Verbände im Vergleich zu den vorangegangenen Kommandostabsübungen" hätten "merklich festigen“ können. So seien sie dazu in der Lage gewesen, „entsprechend den konkreten Gefechtssituationen zweckmäßige Entschlüsse zu fassen, die Verbände, Truppenteile und Waffengattungen im Rahmen einer verbündeten Gruppierung richtig einzusetzen, das Zusammenwirken zwischen ihnen zu organisieren und die Truppen im Verlaufe der Kampfhandlungen fest und ununterbrochen zu führen" 43 . Insgesamt sei eine "positive Entwicklung" in der Gefechtsausbildung der NVA zu verzeichnen ${ }^{44}$. Der Nationale Verteidigungsrat wies jedoch auf die "teilweise noch bestehenden Unterschiede im Ausbildungsstand" der einzelnen Warschauer Pakt-Streitkräfte hin ${ }^{45}$. Unter anderem müßten die Offiziere der NVA noch

39 Bericht über die in der Zeit vom 11. bis 15. September 1963 durchgeführte Übung „Quartett“, 18. Januar 1964, BA-MA, VA-01/12946, Bl. 99.

40 Armee für Frieden und Sozialismus, S. 137.

41 Haack, Zur Rolle sowjetischer Erfahrungen, S. 18.

42 Aus der Ansprache des Oberkommandierenden der Vereinten Streitkräfte der Länder des Warschauer Vertrages, Marschall der Sowjetunion A. A. Gretschko, anläßlich des Empfanges des Staatsrates der DDR zu Ehren der gemeinsamen Truppenübung „Quartett“, 15. September 1963, in: Hanisch, Die NVA in der sozialistischen Verteidigungskoalition, S. $123 \mathrm{f}$.

43 Bericht über die in der Zeit vom 11. bis 15. September 1963 durchgeführte Übung „Quartett", 18. Januar 1964, BA-MA, VA-01/12946, Bl. 102.

44 Ebenda, Bl. 104.

45 Beschluß des Nationalen Verteidigungsrates der DDR vom 20. September 1963 zu den Ergebnissen der Übung „Quartett“. Protokoll der 16. Sitzung des Nationalen Verteidigungsrates der DDR, 20. September 1963, BA-MA, DVW 1/39473, BI. 14. 
intensiver die russische Sprache erlernen und diese auch in der Dienstdurchführung anwenden ${ }^{46}$.

1963 wurden der NVA-Führung im Anschluß an eine zweiseitige strategische Kartenübung auf dem „Westlichen Kriegsschauplatz“, die in der DDR unter der Leitung des sowjetischen Verteidigungsministers durchgeführt worden war, schließlich auch die operativen Planungsdokumente für einen zukünftigen Einsatz der NVA übergeben. Sie enthielten unter anderem die Aufgaben, die die DDRStreitkräfte im Rahmen der Ersten Strategischen Staffel übernehmen sollten, und die in diesem Fall bestehenden Unterstellungsverhältnisse ihrer Verbände ${ }^{47}$.

Der Prozeß der Eingliederung der NVA in die Erste Strategische Staffel fand seinen Abschluß durch das Manöver "Oktobersturm“, das vom 16. bis 22. Oktober 1965 im Südwesten der DDR stattfand ${ }^{48}$. Beteiligt waren wiederum die NVA, die GSSD, die Polnische Armee und die Tschechoslowakische Volksarmee mit Stäben und Truppen. Die Bedeutung, die das Manöver in dieser Hinsicht offenbar hatte, verdeutlicht zum einen die Tatsache, daß neben Ulbricht und Honecker der Oberkommandierende der Vereinten Streitkräfte sowie auch die Verteidigungsminister der Warschauer Pakt-Staaten anwesend waren ${ }^{49}$. Zum anderen mußte die NVA hier den Nachweis erbringen, daß sie nunmehr dazu fähig war, „unter realen“ Einsatzbedingungen zu operieren, denn das Manöver wurde "in unbekanntem Gelände“ ohne die Nutzung von Truppenübungsplätzen durchgeführt ${ }^{50}$.

Der Stellenwert des Manövers läßt sich auch an der gegenüber 1963 erneut gestiegenen Anzahl der eingesetzten Mittel ablesen. Insgesamt nahmen 64100 Armeeangehörige an der Übung teil, davon gehörte beinahe ein Drittel zur NVA. Ferner kamen unter anderem 1204 Panzer, 1370 Schützenpanzer, $10600 \mathrm{Kraftfahrzeuge}$ und 722 Flugzeuge zum Einsatz ${ }^{51}$. Geleitet wurde die Übung vom Oberkommandierenden der GSSD, Armeegeneral Petr K. Koschewoi. Als Befehlshaber einer der beiden im Manöver gegeneinander antretenden Seiten wurde der Chef des Militärbezirkes III Generalmajor Hans Ernst eingesetzt.

Dem Manöver lag das Ziel zugrunde, die Gefechtsbereitschaft und den integrierten Einsatz der verbündeten Armeen in der Anfangsperiode eines Krieges im mitteleuropäischen Raum zu erproben ${ }^{52}$. Überprüft werden sollte, inwieweit die Stäbe und Truppen eines Teils der Ersten Strategischen Staffel dazu in der Lage waren,

46 Ebenda, Bl. 16.

47 Wenzke, Die Nationale Volksarmee, S. 451.

481965 hatten zudem zwei weitere Übungen stattgefunden, die in dieser Hinsicht von Bedeutung waren. Zum einen handelte es sich um eine gemeinsame Truppenübung von Land- und Luftstreitkräften der NVA und der GSSD westlich von Berlin, die vom 5. bis 11. April 1965 stattfand. Bei dieser Übung wurden sowjetische Luftlandetruppen aus einer Entfernung von 1200 Kilometern herangeführt, die die Einheiten der Landstreitkräfte bei der Überwindung großer Wasserhindernisse unterstützen sollten. Die zweite Übung hieß „Zenit-65“ und dauerte vom 29. bis 31. Juli 1965. An ihr waren Luftverteidigungskräfte aller Mitgliedsstaaten des Warschauer Paktes beteiligt. Zeittafel zur Militärgeschichte, S. 199, 205.

49 Zöbisch, Manöver „Oktobersturm“ - militärisches Examen im Herbst 1965, S. 431.

50 Erfahrungen aus dem gemeinsamen Manöver „Oktobersturm“, 6. November 1965, VA-01/18882, Bl. 91.

51 Analyse über das gemeinsame Manöver „Oktobersturm“, 1966, BA-MA, VA-01/1885, Bl. 10.

52 Erfahrungen aus dem gemeinsamen Manöver „Oktobersturm“, 6. November 1965, BA-MA, VA-01/18882, Bl. 93. 
einen Angriff der NATO-Streitkräfte auf dem eigenen Territorium abzuwehren ${ }^{53}$. Dabei ging man erstmals davon aus, daß zunächst nur konventionelle Waffen zum Einsatz kommen würden ${ }^{54}$. Die Truppen der Ersten Strategischen Staffel übten Rückzugs-, Verteidigungs-, Begegnungs- und Angriffsgefechte. Im Rahmen der Angriffshandlungen erhielten die Verbände Unterstützung durch Luftlandetruppen, die über mehrere hundert Kilometer herangeführt wurden und im Rücken des angenommenen Gegners landeten ${ }^{55}$.

Im Ergebnis hieß es von Seiten der NVA, die Übung habe dazu beigetragen, ein einheitliches Niveau der Gefechtsbereitschaft herzustellen sowie das Zusammenwirken und die einheitliche Truppenführung der Streitkräfte des Warschauer Vertrages zu vervollkommnen ${ }^{56}$. Auf der 11. ZK-Tagung im Dezember 1965 äußerte der Chef der Politischen Hauptverwaltung, Waldemar Verner, das Manöver sei „eine echte Überprüfung der Ergebnisse des Ausbildungsjahres 1964/65 in den Armeen der ersten strategischen Staffel“ gewesen. Abschließend stellte er fest: „Ich kann heute dem Zentralkomitee berichten, daß die an dem Manöver, Oktobersturm' beteiligten Truppen der Nationalen Volksarmee erneut bewiesen haben, daß die NVA eine moderne Armee ist, bereit und fähig, jede ihr im Rahmen des Warschauer Vertrages übertragene Aufgabe zu erfüllen. “57

\section{Die Funktion der NVA im Innern}

Obwohl im Zuge der Integration der NVA in die Erste Strategische Staffel des Warschauer Paktes der militärische Einsatz im Bündnis eindeutig im Vordergrund stand, war weiterhin eine Funktion der Armee im Innern des Staates vorgesehen. Die Streitkräfte waren zwar entsprechend dem Beschluß des Nationalen Verteidigungsrates vom 6. April 1962, der alle anderslautenden früheren Bestimmungen aufhob, „für den Einsatz im Innern ... nicht einzuplanen“. Nach dem Mauerbau sollten bei Aufständen oder inneren Unruhen vielmehr vorrangig die „bewaffneten Kräfte des Ministeriums des Innern und die Kampfgruppen der Arbeiterklasse“ zum Einsatz kommen, um „die Sicherheit der Deutschen Demokratischen Republik zu gewährleisten" 58 . Andererseits fixierte der Beschluß aber ausdrücklich, die NVA habe „bereit zu sein, auf besonderen Befehl kurzfristig Aufgaben im Innern ... zu erfüllen“59.

53 Analyse über das gemeinsame Manöver „Oktobersturm“, 1966, BA-MA, VA-01/1885, B1. 15.

54 Zöbisch, Manöver „Oktobersturm“ - militärisches Examen im Herbst 1965, S. 431.

55 Voerster, Die Aprilübung 1965 und das Manöver „Oktobersturm“, S. 176.

56 Erfahrungen aus dem gemeinsamen Manöver „Oktobersturm“, 6. November 1965, BA-MA, VA-01/18882, Bl. 93.

57 Stenografisches Protokoll der 11. Tagung des ZK der SED, 15.-17. Dezember 1965, SAPMO-BArch, DY 30/IV 2/1/336, Bl. 165.

58 Organisierung des inneren Einsatzes. Protokoll der 10. Sitzung des Nationalen Verteidigungsrates der DDR, 6. April 1962, BA-MA, DVW 1/39467, Bl. 4 f. Vgl. Steike, Von den „Inneren Truppen“ zur Bereitschaftspolizei (1953-1990), S. 78 ff.; Wagner, Die Kampfgruppen der Arbeiterklasse (1953-1990), S. 294 ff.

59 Organisierung des inneren Einsatzes. Protokoll der 10. Sitzung des Nationalen Verteidigungsrates der DDR, 6. April 1962, BA-MA, DVW 1/39467, Bl. 4. 
Diese Festlegungen wurden durch einen weiteren Beschluß des Nationalen Verteidigungsrates vom 14. September 1962 zusätzlich konkretisiert: Der Einsatz der Streitkräfte im Innern konnte demnach nur auf Befehl des Vorsitzenden des Nationalen Verteidigungsrates erfolgen. In diesem Fall sollten „operative Gruppen“ der eingesetzten Armeeeinheiten zu den entsprechenden Bezirkseinsatzleitungen kommandiert werden. Gleichzeitig wurden ,alle zur Erfüllung der gestellten Aufgaben eingesetzten Kräfte" dem jeweiligen NVA-Kommandeur unterstellt ${ }^{60}$.

Ein möglicher Einsatz der NVA im Innern gewann ab 1963 an Bedeutung. Offenbar hatte die NVA-Führung Kenntnis über Planungen der Bundeswehr zur Führung des sogenannten „verdeckten Kampfes“ erlangt, der auch als "verdeckter Krieg" bezeichnet wurde61. Diese strategische Konzeption war nach Angaben von Hoffmann im Rahmen der 1963 und 1964 durchgeführten NATO-Kommandostabsübungen „Lion vert" und „Fallex 64" auch in der Praxis „erstmalig in großem Umfang erprobt" worden. Deshalb müsse man nunmehr "mit der Anwendung von Formen des ,verdeckten Kampfes' gegen die sozialistischen Staaten rechnen“, so der Minister ${ }^{62}$.

Hoffmann äußerte die Ansicht, der „verdeckte Krieg“ als Variante der Kriegsführung sei von der Bundeswehr wegen ihrer Schwierigkeiten „bei der Verwirklichung der atomaren Konzeption“ entwickelt worden. Das Ziel seien dabei „begrenzte Aktionen gegen die DDR oder andere sozialistische Staaten notfalls im Alleingang" 63 . Er konstatierte, die „Kriegsart" sei „in jüngster Vergangenheit" neu aufgetaucht ${ }^{64}$. Die SED hatte bereits in den fünfziger Jahren vermeintliche Bundeswehrplanungen zur Führung „begrenzter Kriege“ als einen Hauptbedrohungsfaktor für die innere und äußere Sicherheit der DDR angesehen ${ }^{65}$.

Entsprechend der internen Definition der Bundeswehrführung, die der NVAFührung anscheinend im Detail bekannt war, handelte es sich beim „verdeckten Kampf" um „eine zum Waffeneinsatz gesteigerte Krisensituation von bürgerkriegsähnlichem Charakter“: Der Angreifer operierte demnach jedoch „nicht mit Großverbänden“, sondern blieb „im Kräfteeinsatz unterhalb der Schwelle des konventionellen Krieges" und wandte die „Kampfgrundsätze des Untergrund- und Bandenkampfes" an ${ }^{66}$.

Als Hauptmethode der Kriegsführung wurde gemäß der Bundeswehrplanung „das Einschleusen von Einsatzgruppen in das Hinterland des Gegners mit dem Ziel, aus der dortigen Bevölkerung Banden aufzubauen, diese auszubilden, zu versorgen

60 Grundsätze der Zusammenarbeit zwischen Dienststellen und Einrichtungen des Ministeriums für Nationale Verteidigung mit den bewaffneten Kräften des Ministeriums des Innern. Protokoll der 12. Sitzung des Nationalen Verteidigungsrates der DDR, 14. September 1962, BA-MA, DVW 1/39469, BI. 32.

61 7. Tagung des ZK der SED, 2.-5. Dezember 1964, SAPMO-BArch, DY 30/IV 2/1/319, Bl. 177.

62 Ebenda, Bl. 179.

${ }^{63}$ Konzeption für nächste militärische und politisch-ideologische Maßnahmen. Hoffmann an Ulbricht, 4. November 1964, BA-MA, AZN/32618, Bl. 6.

64 7. Tagung des ZK der SED, 2.-5. Dezember 1964, SAPMO-BArch, DY 30/IV 2/1/319, Bl. $176 \mathrm{ff}$.

$65 \mathrm{Vgl}$. Kap III.1. dieser Studie.

66 7. Tagung des ZK der SED, 2.-5. Dezember 1964, SAPMO-BArch, DY 30/IV 2/1/319, Bl. 177. 
und ihren Kampf zu führen“, betrachtet. Die Aktionen der Banden sollten demzufolge „die Operationsfreiheit und Versorgung der gegnerischen Truppen lähmen und durch Sabotage und Terror ein allgemeines Chaos hervorrufen" 67 .

Die SED ging davon aus, daß der „,verdeckte Krieg' ... mit dem Ziel des Sturzes der bestehenden Machtverhältnisse, der Eroberung eines Teils des fremden Territoriums, der Einsetzung einer dem Angreifer hörigen Regierung oder der Erschütterung der gesellschaftlichen Verhältnisse" geführt werden würde. Dabei wurde unterstellt, die westdeutsche Seite werde den Konflikt als eine allein „innerstaatliche Angelegenheit" bezeichnen ${ }^{68}$. Man ging davon aus, daß letztlich die Absicht bestand, es „konterrevolutionären Kräften“ im Rahmen eines derartigen Aggressionsszenarios zu ermöglichen, „die Macht zu übernehmen und sich unter den Schutz der später angreifenden Streitkräfte zu stellen" 69 .

Aufgrund dieser Bewertung des ,verdeckten Krieges“ konnte die SED Spannungen und Unruhen im Innern der DDR anstatt als isoliertes innenpolitisches Phänomen immer als erste Phase einer westlichen Aggression begreifen, womit sie einmal mehr dem Erklärungsmuster aus den fünfziger Jahren folgte. Ein Militäreinsatz war somit von vornherein gerechtfertigt, auch wenn es noch nicht zu einem direkten Eingreifen von regulären oder irregulären Einheiten der Bundeswehr gekommen war. In diesem Zusammenhang bleibt jedoch unklar, ob die SED-Führung dieses Angriffsszenario für sehr wahrscheinlich hielt oder ob die Planungen der Bundeswehr, deren Verwirklichung im übrigen nach wie vor wenig realistisch war, intern als Legitimation verwandt wurden, den möglichen Einsatz der Streitkräfte im Innern wieder stärker in den Blick zu nehmen. Die NVA war aber nach Einschätzung Hoffmanns „,ür den Einsatz in einem ,verdeckten Krieg' gegen, irreguläre Einhei-

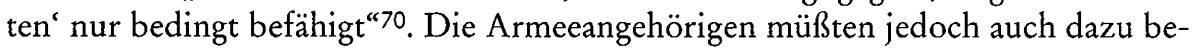
reit sein, „kompromißlos gegen Banden und Diversantengruppen sowie gegen eingedrungene reguläre oder nichtreguläre militärische Einheiten“" zu kämpfen ${ }^{71}$.

Es war vermutlich auch ein Ergebnis ihrer immer noch überspitzten Bedrohungsperzeption, daß die SED der Strategie des „verdeckten Krieges“ noch Mitte der sechziger Jahre eine derartige Bedeutung beimaß. Die UdSSR gelangte jedoch hinsichtlich der Wahrscheinlichkeit eines auf diese Weise eingeleiteten Angriffs des Westens oder der Bundesrepublik gegen die DDR zu einer abweichenden Einschätzung. Hoffmann hatte offenbar den Oberkommandierenden der Vereinten Streitkräfte Gretschko über die seiner Auffassung nach existierenden Vorbereitungen der

67 Ebenda, Bl. 178.

68 Bombrich: „Die politisch-ideologische Diversion als zielgerichtete Tätigkeit in der Konzeption des ,verdeckten Krieges' gegen die Grenzbevölkerung an der Staatsgrenze West im Bezirk Schwerin. Die Beachtung des Zusammenhanges zwischen der politisch-ideologischen Diversion und des, verdeckten Krieges' als Voraussetzung einer erfolgreichen Abwehrarbeit“. Diplomarbeit, 15. November 1966, BStU, ZA, JHS MF 265, Bl. 3.

69 Amelung: „Die Vorbereitung des, verdeckten Krieges' gegen die DDR durch das Bundeskanzleramt (BKA), das Bundesverteidigungsministerium (BMVtdg) und das Bundesinnenministerium (BMdI) und einige Schlußfolgerungen zur rechtzeitigen Aufklärung und Verhinderung dieser Pläne durch das MfS“. Diplomarbeit, 31. Mai 1966, BStU, ZA, JHS MF $258, \mathrm{Bl} .2$.

70 Konzeption für nächste militärische und politisch-ideologische Maßnahmen. Hoffmann an Ulbricht, 4. November 1964, BA-MA, AZN/32618, Bl. 7.

71 Ebenda, Bl. 9. 
„aggressiven Kreise des Westens auf den ,verdeckten Kampf'“ gegen die DDR und die anderen sozialistischen Staaten informiert. Gretschko erwiderte darauf, er teile zwar Hoffmanns „Beunruhigung in dieser Frage“, nehme zugleich aber an, „daß die Organisation und Führung des, verdeckten Kampfes' gegen die DDR unter den gegenwärtigen Bedingungen ohne die Organisation eines größeren militärischen Grenzkonfliktes nicht möglich“ sei. Sollte es der Westen dennoch „entgegen dem gesunden Menschenverstand versuchen, irgendwelche Aktionen feindlicher Elemente innerhalb des Landes zu provozieren“, würden diese „innerhalb kurzer Zeit durch die inneren Kräfte der DDR mit Hilfe der GSSD zerschlagen werden"72.

Parallel zu der offenkundig auf eine Beruhigung der SED-Führung zielenden Stellungnahme erklärte die UdSSR aber ihre Bereitschaft, im Hinblick auf mögliche innere Unruhen weitergehende Planungen anzustellen. Offenbar kam man daraufhin unter anderem überein, die „sowjetischen Truppen zur Liquidierung feindlicher Aktionen nur auf Bitten" der DDR-Regierung heranzuziehen. Die Formulierung beschrieb eine Lage, in der die Kräfte des DDR-Innenministeriums und der NVA für diese Aufgabe nicht mehr ausreichten ${ }^{73}$. Im Ergebnis bestätigte der Nationale Verteidigungsrat am 26. Januar 1966 den „Plan des Einsatzes der bewaffneten Kräfte der Deutschen Demokratischen Republik und der Kräfte der Gruppe der sowjetischen Streitkräfte in Deutschland zur Vereitelung des bewaffneten Eingriffs des Gegners in das staatliche und gesellschaftliche Leben der Deutschen Demokratischen Republik und zur Zerschlagung seiner bewaffneten Gruppierungen und Banden“. Er trat am 15. April 1966 in $\mathrm{Kraft}^{74}$.

\section{Konsolidierung, Autonomiegewinn und Leistungssteigerung des Militärs}

Unmittelbar vor der Abriegelung der DDR am 13. August 1961 stellte sich die Lage der NVA durchaus zwiespältig dar. Zwar war es gelungen, innerhalb von gut fünf Jahren eine moderne Militärorganisation aufzubauen, die in einem großen Maße von der SED kontrolliert wurde. Andererseits aber konnten der innere Zustand und die erreichte Leistungsfähigkeit der Streitkräfte nicht zufriedenstellen. Vor dem Hintergrund, daß die NVA 1961 am Beginn einer weiteren Modernisierung stand und im Rahmen der Integration in die Erste Strategische Staffel des Warschauer Paktes zunehmend auch Bündnisverpflichtungen übernehmen sollte, befand sich das Militär der DDR vor dem Mauerbau in einer durchaus kritischen Phase.

Vor allem die inneren Verhältnisse in der Truppe waren teilweise noch immer desolat ${ }^{75}$. So stieg etwa die Zahl der Bestrafungen, die 1959 bei 59000 gelegen hatte, 1960 auf fast 62000 an. Dabei wurden 15000 Arreststrafen verhängt, die sich zu-

72 Gretschko an Hoffmann, 12. Januar 1965, BA-MA, AZN/28039, Bl. 1.

73 Ebenda, Bl. 2.

74 Plan des Einsatzes der bewaffneten Kräfte der DDR und der Gruppe der sowjetischen Streitkräfte in Deutschland zur Sicherstellung der inneren Ordnung im Verteidigungsfall. Protokoll der 25. Sitzung des Nationalen Verteidigungsrates der DDR, 26. Januar 1966, BA-MA, DVW 1/39482, Bl. 2 f.

75 Vgl. Kap. VI. dieser Studie. 
sammen auf insgesamt 59602 Tage summierten ${ }^{76}$. Diese Entwicklung hielt auch 1961 weiter an ${ }^{77}$. Bei der Zahl der Fahnenfluchten war ebenfalls kein Rückgang zu verzeichnen, im Gegenteil. Laut einem Bericht der Politischen Verwaltung kam es bis zum 13. August $1961 \mathrm{zu}$ einem „Anstieg gegenüber dem gleichen Zeitraum des Jahres 1960 um 40\%“78. Von Seiten der Partei äußerte man, dies müsse „als sehr ernst eingeschätzt werden"79.

Zudem beurteilte die SED die Einsatzbereitschaft der NVA nach wie vor als nicht ausreichend. Verteidigungsminister Hoffmann meinte hierzu in einem kritischen Rückblick: „Bei den erfolgreich bestandenen Bewährungsproben der Nationalen Volksarmee im Jahre 1961 sind zugleich ernste Mängel in der Arbeit der Kommandeure, Stäbe und Politorgane, in der politischen und militärischen Truppenführung, in der Versorgung und Betreuung der Truppen sowie in der militärischen Disziplin und Ordnung zutage getreten." Eine Reihe von Maßnahmen und Entschlüssen zeugte seiner Ansicht nach unter anderem von „veralteten Auffassungen in Fragen der Kriegskunst und Lücken und Rückständen in der Ausbildung" 80 .

Besonders besorgniserregend war für die SED jedoch das mangelhafte Leistungsvermögen der Offiziere. Zwar hatte die Partei weitgehend ihr Ziel erreicht, ein neues Offizierskorps zu schaffen. Anfang 1961 kamen nach Angaben der ZK-Abteilung für Sicherheitsfragen 79,0 Prozent der Offiziere aus der Arbeiterschaft, 3,7 Prozent waren ihrer sozialen Herkunft nach Bauern ${ }^{81}$. Auch glaubte die Partei aufgrund der Tatsache, daß 95,6 Prozent der Offiziere der Partei als Mitglieder oder Kandidaten angehörten ${ }^{82}$, von einem hohen Maß politischer Zuverlässigkeit des Führerkorps der NVA ausgehen zu können. Auf der anderen Seite mußte die SED aber erkennen, daß die bis dahin vorrangig berücksichtigten politischen Kriterien in der Personalpolitik nicht den Effekt gehabt hatten, die Qualität des Offizierskorps nachhaltig zu verbessern. Daher verfügten im Januar 1961 immer noch 67,2 Prozent der Offiziere nur über einen Volksschulabschluß, 4,6 Prozent hatten jedoch noch nicht einmal diesen erreicht. Nur 12,1 Prozent der Offiziere besaßen die Mittlere Reife und 16,1 Prozent das Abitur ${ }^{83}$.

76 Protokoll der Parteiaktivtagung der Parteiorganisationen des Ministeriums für Nationale Verteidigung, 15. Februar 1961, BA-MA, VA-P-01/513, Bl. 11.

77 Bericht über einige Probleme der politisch-ideologischen Erziehungsarbeit und politischmilitärischen Leitungstätigkeit in den Einheiten, Verbänden und Truppenteilen der NVA, 29. November 1962, BStU, ZA, MfS ZAIG 691, Bl. 12.

78 Bericht über den Stand der militärischen Disziplin und Ordnung, 1961, BA-MA, VA-P-01/13499, Bl. 113.

79 Bericht über die Fahnenfluchten in den bewaffneten Kräften der DDR, 8. August 1961, SAPMO-BArch, DY 30/IV 2/12/47, Bl. 231.

80 Die politische Arbeit im Ausbildungsjahr 1962. Direktive Nr. 10/61 des Ministers für Nationale Verteidigung, 8. Dezember 1961, BA-MA, VA-01/6087, Bl. 133. Hoffmanns Einschätzung bezog sich dabei vor allem auf die Leistungen der NVA in Übungen, die im Verlauf des Jahres 1961 stattgefunden hatten. Entwurf von Thesen über die neue Entwicklungsetappe der NVA und die sich daraus ergebenden Aufgaben, 1961, SAPMO-BArch, DY 30/IV 2/12/15, Bl. 149.

81 Auswertung über die Personalstatistik des Offiziersbestandes der NVA, 1. Januar 1962, SAPMO-BArch, DY 30/IV 2/12/29, Bl. 127.

82 Ebenda, Bl. 129.

83 Ebenda, Bl. 137. 
Die Bildungs- und Ausbildungsdefizite ${ }^{84}$ wirkten sich in dieser Phase spürbar nachteilig auf den Leistungsstand der militärischen Truppenführung aus. Hoffmann äußerte im Juni 1961, die Offiziere zeigten unter anderem große Schwächen „in der Beherrschung der taktisch-technischen Daten und Einsatzgrundsätze der modernen Waffen". Zusammenfassend könne man feststellen, so der Minister für Nationale Verteidigung, daß die „operativ-taktischen Kenntnisse und Fertigkeiten der Kommandeure und Stäbe zur Planung und Organisation von Operationen und Gefechten" noch nicht ausreichten, „um alle Fragen schnell, präzise und zweckmäßig zu entscheiden und zu verwirklichen" 85 . Obwohl die Mehrheit der Offiziere keine Anstrengungen scheue, sich auch militärwissenschaftlich weiterzuqualifizieren, bleibe man „im Tempo hinter den Erfordernissen zurück“"86.

Bereits drei Monate zuvor war eine Brigade des ZK bei der Überprüfung der 11. Motorisierten Schützendivision zu dem Ergebnis gekommen, daß das "gegenwärtige Niveau der technischen Qualifizierung und naturwissenschaftlich-technischen Bildung“ weder den aktuellen Aufgabenstellungen, aber „erst recht nicht den zukünftigen Anforderungen“ gerecht werde. Im Bericht der Brigade heißt es: „Es zeigt sich ein Widerspruch zwischen der auf Grund der vorhandenen und zu erwartenden Technik notwendigen naturwissenschaftlich-technischen Bildung und dem derzeitigen vorhandenen Bildungsstand der Offiziere." Während der Untersuchung erklärte eine Reihe von Offizieren, die an den Offiziersschulen vermittelten Ausbildungsinhalte entsprächen „nicht mehr den Forderungen der praktischen Arbeit in der Truppe“. Unter anderem würden dort „völlig ungenügende technische Kenntnisse vermittelt" ${ }^{\text {" }}$.

Zudem trat fünf Jahre nach Gründung der NVA nunmehr deutlich zutage, daß diejenigen hohen Parteifunktionäre in militärischen Führungspositionen, die sich vorwiegend aus den Reihen ehemaliger Funktionäre der KPD (Kommunistische Partei Deutschlands) rekrutierten und vor allem aus politischen Erwägungen von der SED-Führung in ihre Dienststellungen eingesetzt worden waren, jetzt offensichtlich an ihre Leistungsgrenze stießen. Die ZK-Abteilung für Sicherheitsfragen äußerte diesbezüglich: „Alte[,] verdienstvolle Genossen in der Arbeiterbewegung in leitenden Funktionen der Armee geraten in Gefahr, sich ernste gesundheitliche Schäden zuzuziehen oder in einigen Fällen sich der Lächerlichkeit preiszugeben, das Ansehen und Vertrauen zu verlieren, wenn man sie weiter leichtfertig in verantwortliche[n] Funktionen, die ihre Fähigkeiten übersteigen[,] beläßt. Diese Genossen haben nicht verdient, daß sie wegen Mängel[ $n]$ in ihrer Arbeit schuldlos der Kritik ausgesetzt sind, bzw. sich Genossen niedriger Dienstgrade nicht selten über sie lustig machen." 88

84 Vgl. Kap. III.3. dieser Studie.

85 Protokoll der III. Delegiertenkonferenz der Parteiorganisationen der SED in der NVA, 10./11. Juni 1961, BA-MA, VA-P-01/557, Bl. $209 \mathrm{f}$.

86 Ebenda, Bl. 214.

87 Bericht der Brigade des ZK der SED in der 11. Mot. Schützendivision der NVA, 1961, SAPMO-BArch, DY 30/IV 2/12/24, Bl. $58 \mathrm{ff}$.

88 Außerplanmäßige Analyse über den Zustand der Kaderarbeit in der NVA, 1961, SAPMOBArch, DY 30/IV 2/12/29, Bl. 301. So konstatierte man zum Beispiel, dem Politstellvertreter der Militärakademie „Friedrich Engels“ Generalmajor Paul Blechschmidt fehlten für diese Tätigkeit „jegliche Fähigkeiten“ und der Abteilungsleiter Schulen in der Verwaltung für Ausbildung Generalmajor Dollwetzel sei „vollkommen krank und für eine Funktion in der 
Der SED-Führung dürfte spätestens zu diesem Zeitpunkt klar geworden sein, daß die Qualität eines großen Teils des Offizierskorps der NVA nicht mehr ausreichte, um in Zukunft insbesondere die Anforderungen zu erfüllen, die aus der Eingliederung der Streitkräfte in die Erste Strategische Staffel des Warschauer Paktes erwuchsen. Daher erwies sich unter anderem eine grundlegende Neuorientierung in der Kaderpolitik als zwingend notwendig. Die Leitung des Ministeriums für Nationale Verteidigung stellte im Juni 1961 in Übereinstimmung damit fest: „Die komplizierte militärpolitische Lage in Deutschland, die neue Entwicklungsetappe der Nationalen Volksarmee verlangen eine höhere politische, militärische und militärtechnische Qualifizierung aller Offiziere."89

Ein weiteres, schwerwiegendes Problem, das sich der SED 1961 stellte, war der akute Personalmangel. Aufgrund der Tatsache, daß die Werbung für den freiwilligen Dienst in der NVA nun vor allem unter den im Krieg geborenen Jugendlichen erfolgen mußte, entstand ein massiver Engpaß. Während in der DDR der Geburtsjahrgang 1940 noch 145.914 männliche Jugendliche umfaßte, sank die Zahl des Jahrganges 1945 auf $76084 \mathrm{ab}$. Bis zum April 1961 konnten statt der angestrebten 17400 Jugendlichen nur 9724 für den Dienst in den Streitkräften geworben werden ${ }^{90}$. So mußte etwa der Chef des Militärbezirkes III Kunath Ende März 1961 an Hoffmann melden, die vom Chef des Hauptstabes gestellten Aufgaben für die Werbung seien in seinem Militärbezirk bisher „nur unzureichend erfüllt" worden. Auch die bis Dezember 1960 erreichten Ergebnisse seien „völlig unbefriedigend“91. Letztlich gelang es zwar anscheinend doch noch, das notwendige Personal zu rekrutieren. Vermutlich erkannte die SED-Führung jedoch bereits Anfang 1961, daß dies auf der Grundlage des Freiwilligenprinzips im folgenden Jahr nicht noch einmal zu bewerkstelligen sein würde. Ulbricht räumte dann auch im Dezember 1961 gegenüber Chruschtschow ein, die Wiederholung der „allgemeinen Kampagne“, mit der man die NVA in diesem Jahr aufgefüllt habe, sei „nicht möglich"92.

Nicht zuletzt in der Frage der Nachwuchsgewinnung zeigte sich, daß eine Stabilisierung der DDR-Streitkräfte unerläßlich geworden war. Dies jedoch nicht nur, um die zukünftigen Aufgaben besser erfüllen zu können, sondern allein schon, um den bisher erreichten Entwicklungsstand zu halten. Anfang 1961 befand sich das Militär dabei in einer ähnlichen Situation wie die DDR im ganzen. Die Flucht vor

Armee ungeeignet". Zudem befand die ZK-Abteilung für Sicherheitsfragen, die Kommando-Stabsübung Ende Mai 1961 habe "deutlich bestätigt", daß unter anderem die Chefs der Militärbezirke III und V, die Generalmajore Martin Bleck und Artur Kunath, „keine Kommandeure einer modernen Armee werden“ könnten. Daraus folgerte man schließlich, es könne zu „ernsten Schwierigkeiten in der Führung der Armee und der Erhaltung der Gefechtsbereitschaft kommen", wenn keine Konsequenzen hinsichtlich der zukünftigen Besetzung vieler leitender Funktionen gezogen würden. Ebenda, Bl. $302 \mathrm{f}$.

89 Konzeption zur Heranbildung politisch und militärisch hochqualifizierter Offiziere und zur Sicherung des Nachwuchses sowie der Qualifizierung von Politoffizieren. Protokoll der Sitzung der Leitung des Ministeriums für Nationale Verteidigung, 9. Juni 1961, BA-MA, AZN/28076, Bl. 102.

90 Patzer, Die personelle Auffüllung der NVA, S. 366.

91 Mitteilung des Chefs des Militärbezirkes III Generalmajor Kunath an Hoffmann, 27. März 1961, BA-MA, VA-01/6144, Bl. 2 f.

92 Ulbricht an Chruschtschow, 13. Dezember 1961, SAPMO-BArch, DY 30/J IV 2/202/66, Bl. 134. 
allem junger Menschen in die Bundesrepublik - 50 Prozent der Flüchtlinge waren unter 25 Jahre alt - führte nicht nur dazu, daß in der DDR ein allgemeiner Arbeitskräftemangel herrschte, der eine der Hauptursachen für die Krise des SED-Regimes war93. Die geflohenen männlichen Jugendlichen konnten natürlich auch von der NVA nicht mehr rekrutiert werden. Die Abriegelung der DDR war insofern auch für die NVA von herausragender Bedeutung. Letztlich war sie die Voraussetzung für eine kontinuierliche und erfolgreiche Weiterentwicklung der Streitkräfte.

Zudem dürfte sich die SED darüber im klaren gewesen sein, daß eine tiefgreifende Verbesserung der Einsatzbereitschaft und die Steigerung der Leistungsfähigkeit des Militärs nur durch die Einführung der allgemeinen Wehrpflicht zu erreichen waren. Hierzu äußerte sich Ulbricht nach dem Mauerbau unmißverständlich: „Würden wir die allgemeine Wehrpflicht nicht einführen, so wäre die Auffüllung der Nationalen Volksarmee ... entsprechend den Verpflichtungen des Warschauer Vertrages nicht mehr voll gewährleistet." Neben der Beseitigung des Rekrutierungsproblems erhoffte er sich von der Maßnahme, eine bessere Auswahl des Personals „nach politischer Zuverlässigkeit und fachlicher Qualifikation" erreichen zu können ${ }^{94}$. Die Wehrpflicht aber ließ sich ebenfalls erst nach der Abriegelung der DDR einführen, anderenfalls wäre es wohl zu einer noch größeren Fluchtbewegung männlicher Jugendlicher in den Westen gekommen ${ }^{95}$. Auch aus diesem Grund war der Bau der Mauer eine conditio sine qua non.

Unmittelbar im Anschluß an den 13. August 1961 trat die NVA in eine Phase der Konsolidierung ein. Die Abgrenzung der DDR bewirkte eine Verminderung des vielfältigen Drucks, der als Folge der offenen Grenze auch auf dem Militär gelastet hatte. Im Zuge der veränderten Rahmenbedingungen eröffnete sich der SED jetzt die Möglichkeit, ihre militärpolitischen Ziele neu zu formulieren. Diesen Prozeß hatte sie bereits Mitte 1961 sowohl theoretisch als auch praktisch eingeleitet. Nach dem Mauerbau konnte die Partei die Verwirklichung der Zielsetzungen verstärkt in Angriff nehmen.

Nachdem die politischen Strukturen und Mechanismen zur Kontrolle und Steuerung der militärischen Entscheidungsprozesse bis zu diesem Zeitpunkt umfassend etabliert worden waren, war es der Partei möglich, von der Praxis einer extensiven politischen Durchdringung der NVA abzugehen. Sie beschränkte sich zunehmend auf eine übergeordnete Kontrolle der Truppenführung. Alle grundlegenden militärpolitischen Entscheidungen wurden jedoch weiterhin uneingeschränkt vom Politbüro gefällt ${ }^{96}$. Durch diese Entwicklung erlangte die Truppenführung der NVA ein gewisses Maß an fachlicher Autonomie. Dies bedeutete eine Zunahme der Selbstorganisationsfähigkeit des Militärs. Fachspezifische Kriterien traten bei der Bewertung militärischer Fragen zunehmend in den Vordergrund. So wurde die militärische Einsatzbereitschaft, die vor allem im Hinblick auf die Integration der NVA in die Erste Strategische Staffel an Wichtigkeit gewann, nicht mehr zuerst an politischen Maßstäben gemessen. Vielmehr verlor der politische Aspekt mehr und mehr

93 Vgl. Weber, Die DDR 1945-1990, S. 58.

94 Ulbricht an Chruschtschow, 13. Dezember 1961, SAPMO-BArch, DY 30/J IV 2/202/66, Bl. 135.

95 Vgl. Froh, Sechs Jahre war sie im Warschauer Pakt, S. 8.

96 Vgl. Protokoll Nr. 25/65 der außerordentlichen Sitzung des Politbüros des ZK der SED, 25. Juni 1965, SAPMO-BArch, DY 30/J IV 2/2/993, Bl. $1 \mathrm{ff}$. 
an Bedeutung. Ausschlaggebend bei der Bewertung der Leistungsfähigkeit der Streitkräfte waren jetzt in erster Linie der Grad ihrer Technisierung und des operativen Leistungsvermögens sowie die Qualifikation des Personals. Diesen Prioritätenwechsel in der Militärpolitik der SED ließ bereits ein vom Stellvertreter des Ministers für Nationale Verteidigung für Technik und Bewaffnung, Dickel, im Juli 1961 veröffentlichter Artikel erkennen. Darin führte er aus, daß das politische Bewußtsein und die Moral der Armeeangehörigen zwar von „entscheidender Bedeutung" seien. Die beste Technik und das höchste Bewußtsein nützten jedoch praktisch nichts, wenn die Soldaten und Offiziere es nicht verstünden, „richtig mit den Waffen und Geräten umzugehen". Daher benötigten vor allem die Offiziere eine „umfassende wissenschaftlich-technische Bildung und ausgezeichnete, konkrete Kenntnisse auf ihrem Fachgebiet“, die sie vor allem auch dazu befähigten, „mit der weiteren technischen Entwicklung Schritt zu halten"97.

Nach dem Mauerbau traten politische Gesichtspunkte im Zusammenhang mit der militärischen Leistungsfähigkeit nunmehr endgültig in den Hintergrund. So erwähnte Hoffmann auf der 14. ZK-Tagung im November 1961 den politischen Aspekt nicht mehr ausdrücklich als einen Schwerpunkt der zukünftigen Militärpolitik. Statt dessen bezeichnete er die „Heranbildung hochqualifizierter wissenschaftlich-technischer Kader" sowie „die ständige Vervollkommnung und die Einführung der modernsten Technik" als vorrangig zu lösende Aufgaben".

Im Hinblick auf das favorisierte Anforderungsprofil der NVA-Offiziere setzte die SED-Führung im Gegensatz zu den fünfziger Jahren jetzt anstatt auf politische primär auf fachspezifische Kriterien. Hoffmann konstatierte Anfang 1963, der moderne Krieg verlange „allseitig militärtechnisch gebildete Truppenoffiziere“99. Der Offizier sollte vor allem ein umfassend qualifizierter Militärspezialist sein. Dementsprechend hieß es in einem Papier des Nationalen Verteidigungsrates: „Meisterhafte Beherrschung und Ausnutzung der Technik, schnelles Handeln, Kühnheit, schöpferische Ideen und vernünftige Initiative in der Taktik sowie fester Wille und die Fähigkeit, jede Situation zu meistern, müssen den Kommandeur auszeichnen. " Die Autorität des Vorgesetzten hänge "heute weitgehend von seinen technischen Kenntnissen und der Fähigkeit ab, sie in der Ausbildung sinnvoll und interessant zu vermitteln"100. Diese Beschreibung zeigt, daß das Leitbild des Offiziers in der Realität einer Entpolitisierungstendenz unterlag, die zugleich charakteristisch war für die voranschreitende Professionalisierung der NVA. Politisch konformes Verhalten blieb aber nichtsdestoweniger eine entscheidende Bedingung für das berufliche Fortkommen eines Offiziers.

Proletarische Herkunft und Parteizugehörigkeit allein jedoch verliehen einem Offizier nun keine besondere Autorität mehr. Auch besaßen beide Kriterien in der

${ }^{77}$ Dickel, Die Notwendigkeit einer allseitig wissenschaftlich-technischen Qualifizierung, S. 867.

98 Aus der Diskussionsrede des Mitglieds des ZK der SED und Ministers für Nationale Verteidigung der DDR, Armeegeneral H. Hoffmann, auf der 14. Tagung des ZK der SED, 23.-26. November 1961, in: Die Militär- und Sicherheitspolitik der SED, S. 294.

99 Hoffmann, Wie erreichen wir in der Armee, S. 173.

100 Probleme der Erziehung und Bildung in der NVA, die sich aus der modernen Militärtechnik ergeben. Protokoll der 15. Sitzung des Nationalen Verteidigungsrates der DDR, 13. Juni 1963, BA-MA, DVW 1/39472, Bl. 41. 
Personalpolitik kaum mehr gestaltende Kraft. Die Parteimitgliedschaft war nur mehr eine allgemeine Voraussetzung, um als Offizier in höhere Dienststellungen versetzt werden zu können. Sie erreichte im Januar 1962 mit 98,1 Prozent ihren vorläufigen Höchststand ${ }^{101}$. Zumindest formal konnte dieser hohe Prozentsatz als Zeichen einer großen politischen Übereinstimmung des Offizierskorps mit der SED gewertet werden. Die Parteimitgliedschaft lag von jetzt an immer über 95,0 Prozent, variierte jedoch wegen der permanenten $\mathrm{Zu}$ - und Abgänge von Offizieren ${ }^{102}$. So waren Mitte 1963 genau 97,0 Prozent der Offiziere Mitglieder oder Kandidaten der SED ${ }^{103} .1964$ betrug der Anteil 98,0 Prozent ${ }^{104}$, der in der Folgezeit wieder zurückging und 1966 bei 95,6 Prozent lag105.

Die Zugehörigkeit zur Arbeiterklasse wiederum wurde in erster Linie nur noch als ideale Eigenschaft eines Offiziers angesehen. Anstelle der sozialen Herkunft wurde politisches Engagement zunehmend höher bewertet. So hieß es 1962: „Es ist $\mathrm{zu}$ gewährleisten, daß nur solche Offiziersbewerber die Ausbildung zum Offizier beginnen, die politisch zuverlässig und entwicklungsfähig sind sowie durch aktive Teilnahme am gesellschaftlichen Leben und vorbildliche Erfüllung ihrer Pflichten, ihre Verbundenheit zur Arbeiter-und-Bauern-Macht unter Beweis gestellt haben." 106 Vor allem nach dem VI. Parteitag der SED im Januar 1963 verlor die Klassenzugehörigkeit kaderpolitisch weiter spürbar an Gewicht. Ulbricht hatte hier geäußert, in „früheren Abschnitten der Entwicklung“ der DDR seien „Klassenzugehörigkeit und soziale Herkunft besonders beurteilt" worden. Inzwischen habe man jedoch ein Entwicklungsstadium erreicht, das, „ohne die Rolle der Arbeiterklasse zu mindern“, durch "gleiches Recht für alle Bürger auf der Grundlage der Gesetze gekennzeichnet" sei ${ }^{107}$. Dieses Argument erlaubte es in der Folgezeit, auch im Offizierskorps der NVA den Anteil der Angehörigen der sogenannten Intelligenz zu erhöhen, was sich angesichts der fortschreitenden Technisierung der Streitkräfte als unerläßlich erwies. Der Prozentsatz der Offiziere, die nach Angaben der SED aus der Arbeiterklasse stammten, lag daher Ende der achtziger Jahre nur noch bei gut 60 Prozent ${ }^{108}$. In der ersten Hälfte der sechziger Jahre verharrte der Anteil jedoch zunächst auf einem gleichbleibenden Niveau. Anfang 1962 lag er bei 84,7 Prozent ${ }^{109}$.

101 Auswertung über die Personalstatistik des Offiziersbestandes der NVA, 1. Januar 1962, SAPMO-BArch, DY 30/IV 2/12/29, Bl. 129.

102 Fingerle, Waffen in Arbeiterhand?, S. 133.

103 Referat des Ministers für Nationale Verteidigung auf der Kadertagung des Ministeriums für Nationale Verteidigung, 24. Mai 1963, BA-MA, VA-01/13871, Bl. 8.

104 Protokoll Nr. 17/64 der Sitzung der Kreisleitung der SED im Ministerium für Nationale Verteidigung, 3. Januar 1964, BA-MA, VA-P-01/501, Bl. 212.

105 Teilbeitrag der Verwaltung Kader zur Einarbeitung in die Gesamtanalyse und Grundkonzeption der Entwicklung der NVA, 10. März 1967, BA-MA, VA-01/5679, Bl. 17.

106 Grundsätze für die Änderung der politischen und militärischen Erziehungs- und Bildungsarbeit an den Offiziersschulen der NVA, 1962, SAPMO-BArch, DY 30/IV 2/12/32, Bl. 129.

107 Das Programm des Sozialismus, S. 176.

108 Der Prozentsatz der Offiziere, die ihrer Herkunft nach Angehörige der sogenannten Intelligenz waren, lag 1964 bei nur 1,1 Prozent, steigerte sich aber bis 1988 auf 20,2 Prozent. Fingerle, Waffen in Arbeiterhand?, S. 127.

109 Auswertung über die Personalstatistik des Offiziersbestandes der NVA, 1. Januar 1962, SAPMO-BArch, DY 30/IV 2/12/29, Bl. 127. 
1964 waren ebenfalls 84,7 Prozent ihrer sozialen Herkunft nach Arbeiter oder Bauern ${ }^{110} .1966$ lag der Wert dann bei 85,0 Prozent ${ }^{111}$.

Gerade die Änderungen in der Werbung und Personalentwicklung der Offiziere $^{112}$ sowie die auf diese Weise erzielten Ergebnisse verdeutlichen im besonderen Maße die zunehmende Professionalisierung der NVA seit 1961113. Bereits im Juli 1961 äußerte Dickel, alle Offiziere müßten in der Zukunft „ein naturwissenschaftliches und technisches Grundwissen besitzen, das dem von Absolventen einer allgemeinbildenden polytechnischen Oberschule" entspreche ${ }^{114}$. Diese Äußerung deutete den bevorstehenden tiefgreifenden Kurswechsel in der Rekrutierungspraxis an. Die bis dahin verfolgte Linie, den Offiziersnachwuchs unter denjenigen Unteroffizieren und Soldaten zu werben, die ihrer sozialen Herkunft oder ihrer Tätigkeit nach Arbeiter oder werktätige Bauern waren, wie es die Direktive vom 16. August 1958 festgelegt hatte ${ }^{115}$, sollte offensichtlich korrigiert werden. Jetzt konzentrierte sich das Interesse verstärkt auf Jugendliche aus dem zivilen Bereich, die ein entscheidend höheres Bildungs- und Ausbildungsniveau vorweisen konnten. So äußerte beispielsweise Generalmajor Ottomar Pech, der 1961-1979 Chef der Verwaltung Kader war, die „Werbung von geeigneten wissenschaftlich-technischen sowie ökonomischen Kadern“ solle „zum größten Teil für alle Waffengattungen unter den Absolventen von zivilen Hoch- und Fachschulen erfolgen". Grundsätzlich müsse es das Ziel sein, diese Hoch- und Fachschulkader zu Offizieren auszubilden ${ }^{116}$.

Dieser Neuansatz in der Personalpolitik wurde direkt im Anschluß an den Mauerbau durch den Minister für Nationale Verteidigung realisiert. Im Befehl über die „Heranbildung von Offizieren an den Offiziersschulen der NVA“ vom 21. September 1961 heißt es dementsprechend: „Als Bewerber für die Offizierslaufbahn sind Unteroffiziere und Soldaten sowie Bewerber aus dem zivilen Sektor auszuwählen, die das Abitur oder die Kenntnisse der 10. Klasse der allgemeinbildenden polytechnischen Oberschule und nach Möglichkeit eine abgeschlossene Berufsausbildung besitzen." Während Klassenzugehörigkeit als Auswahlkriterium nicht mehr erwähnt wurde, sollte die Rekrutierung jetzt ausdrücklich primär auf der Grundlage der schulischen und beruflichen Qualifikation erfolgen. Solange es aber noch nicht möglich war, den bestehenden Bedarf auf der Grundlage der neuen Vorgaben zu decken, beabsichtigte man übergangsweise, auch noch auf „Unteroffiziere und Sol-

110 Fingerle, Waffen in Arbeiterhand?, S. 127.

111 Teilbeitrag der Verwaltung Kader zur Einarbeitung in die Gesamtanalyse und Grundkonzeption der Entwicklung der NVA, 10. März 1967, BA-MA, VA-01/5679, Bl. 17.

112 Der Offiziersbestand der NVA lag Anfang 1961 bei 21386 Mann. Durch die Eingliederung der Grenztruppen erhöhte er sich auf 25733 im Jahr 1962. 1963 erreichte die Zahl mit 27708 Offizieren den vorläufig höchsten Stand. Ende 1965 hatte die NVA einen realen Bestand von 27149 Offizieren. Der Anteil der Politoffiziere darunter steigerte sich von 2790 Anfang 1961 bis auf 3421 Ende 1965. Stärkenachweisbuch - Gesamtbestand der Offiziere der NVA, Januar 1957-Juni 1967, BA-MA, VA-01/32458, Bl. $18 \mathrm{ff}$.

113 Vgl. Fingerle, Waffen in Arbeiterhand?, S. 124 ff.; Greese/Voerster, Probleme der Auswahl und Förderung der Offizierskader, S. 42 ff.; Herspring, East German Civil-Military Relations, S. $93 \mathrm{ff} ., 120 \mathrm{ff}$.

114 Dickel, Die Notwendigkeit einer allseitig wissenschaftlich-technischen Qualifizierung, S. 870.

115 Vgl. Kap. III.3. dieser Studie.

116 Protokoll der Parteiaktivtagung der Parteiorganisationen der SED in der NVA, 13. Juni 1961, BA-MA, VA-P-01/554, Bl. $72 \mathrm{f}$. 
daten mit erfolgreich abgeschlossener Grundschul- und Berufsausbildung als Bewerber für die Offiziersschulen " zurückzugreifen"117. Diese sollten jedoch zuvor im Rahmen von sechsmonatigen Vorbereitungslehrgängen in den „Hauptzweigen der Allgemeinbildung " die Kenntnisse der 10. Klasse der allgemeinbildenden polytechnischen Oberschule erhalten ${ }^{118}$.

Das angestrebte höhere Bildungsniveau sollte aber nicht zu Lasten fundierter militärischer Grundkenntnisse der Offiziersbewerber gehen. Voraussetzung für den Beginn der Offiziersausbildung war daher, daß der Bewerber zuvor die „allgemeine militärische Grundausbildung, Spezialausbildung und Gruppenausbildung durchlaufen" hatte. Offiziersbewerbern aus dem zivilen Sektor sollten diese Kenntnisse in einem viermonatigen Vorbereitungslehrgang vermittelt werden ${ }^{119}$. Darüber hinaus befahl Hoffmann in Übereinstimmung mit den von Pech drei Monate zuvor geäußerten Überlegungen die Besetzung der vorhandenen Ingenieursplanstellen in den Streitkräften mit "Jungingenieuren aus dem zivilen Sektor". Diese sollten in speziellen Lehrgängen auf ihre „militärtechnischen“ Aufgaben vorbereitet werden. Daneben wollte man auch versuchen, befähigte Offiziersschüler des dritten Lehrjahres in einem weiteren Jahr zum Ingenieur auszubilden ${ }^{120}$.

Der SED gelang es, den Bildungs- und Ausbildungsstand der Offiziere in den nächsten Monaten weiter zu erhöhen. Zwar verfügten Anfang 1962 noch immer 65,9 Prozent der Offiziere nur über eine Volksschulbildung. Der Anteil der Offiziere, die eine weiterführende Schule besucht hatten, stieg jedoch an: 18,5 Prozent besaßen jetzt die Mittlere Reife und 15,6 Prozent das Abitur ${ }^{121}$. In bezug auf die militärische Qualifikation gab es kurzfristig aber keine nachhaltige Verbesserung. Zum 1. Januar 1962 verfügten 10,0 Prozent der Offiziere über eine einjährige, 10,7 Prozent über eine zweijährige, 23,0 Prozent über eine dreijährige und 1,0 Prozent über eine vierjährige Offiziersausbildung. 3,4 Prozent der Offiziere hatten die Militär-

117 Heranbildung von Offizieren an den Offiziersschulen der NVA. Befehl Nr. 66/61 des Ministers für Nationale Verteidigung, 21. September 1961, BA-MA, VA-01/5905, Bl. 22.

118 Ebenda, Bl. 23.

119 Ebenda.

120 Auswahl und Ausbildung der Ingenieurkader für die NVA. Befehl Nr. 63/61 des Ministers für Nationale Verteidigung, 21. September 1961, BA-MA, VA-01/5905, Bl. $7 \mathrm{f}$. Der erkennbare Paradigmenwechsel in der Personalentwicklung des Offizierskorps nach dem 13. August 1961 erzeugte anscheinend bei der Gruppe ehemaliger NVA-Offiziere, die bereits als Offiziere in der Wehrmacht gedient hatten und ab 1957 in die Reserve versetzt worden waren, die Erwartung, für sie könnte sich erneut eine berufliche Perspektive in den Streitkräften eröffnen. Vermutlich in Anbetracht der Tatsache, daß im Zuge der Modernisierung der NVA der Bedarf an gutausgebildeten militärischen Fachleuten weiter anstieg, stellte eine Reihe von ihnen einen „Antrag auf Reaktivierung“. Diese Anträge, wie der des Generalmajors der Reserve Hermann Rentzsch, wurden jedoch mit der Begründung abgelehnt, etwaige Reaktivierungen würden „Zweifellos wieder Angriffspunkt des Gegners zur weiteren Hetze und Verleumdung" der bewaffneten Organe werden. Stellungnahme zur Kadervorlage, 24. November 1961, SAPMO-BArch, DY 30/IV 2/12/29, Bl. 79. 1962 gab es noch 84 ehemalige Wehrmachtsoffiziere in der NVA; 1965 waren es 67. Die meisten von ihnen waren in Ausbildungseinrichtungen tätig. Wenzke, Wehrmachtsoffiziere in den DDR-Streitkräften, S. $152 \mathrm{f}$.

121 Auswertung über die Personalstatistik des Offiziersbestandes der NVA, 1. Januar 1962, SAPMO-BArch, DY 30/IV 2/12/29, Bl. 137. 
akademie und 34,6 Prozent Sonder- und Qualifizierungslehrgänge absolviert, 16,1 Prozent aber noch immer keine militärische Schule besucht ${ }^{122}$.

Eine spürbare Verbesserung der Rahmenbedingungen der Rekrutierung erbrachte die Einführung der allgemeinen Wehrpflicht zum 24. Januar 1962. Das Gesetz verpflichtete alle männlichen Bürger der DDR, mit dem Erreichen des 18. Lebensjahres einen eineinhalbjährigen Grundwehrdienst in der NVA abzuleisten ${ }^{123}$. Infolgedessen war es nun zum einen wesentlich einfacher, geeignete Offiziersbewerber herauszufinden; zum anderen ließen sich Wehrpflichtige leichter als Zivilisten für den Offiziersberuf gewinnen. Bei der Begründung des Wehrpflichtgesetzes äußerte Hoffmann dementsprechend vor der Volkskammer: „In Verbindung mit dem freiwilligen Dienst in der Nationalen Volksarmee wird die allgemeine Wehrpflicht die kontinuierliche Auffüllung unserer Streitkräfte mit Soldaten, Unteroffizieren und Offiziersbewerbern garantieren, die bereits von der Schule und vom Beruf her über polytechnische Kenntnisse verfügen ...."124 Die Erwartungen der Parteiführung erfüllten sich im Hinblick auf die Rekrutierung des Offiziersnachwuchses im Anschluß außerordentlich schnell.

Vor allem die Einführung der Wehrpflicht bewirkte, daß man nach Abschluß der Werbung der Offiziersbewerber für das Jahr 1963 feststellen konnte, es werde schon bald möglich sein, den Offiziersnachwuchs „voll aus Absolventen der erweiterten Oberschulen (Abitur) bzw. der allgemeinbildenden polytechnischen Oberschulen (10 Klassen) auszuwählen" 125 . So entsprachen von den bis Mitte Mai 1962 geworbenen Offiziersbewerbern 75 Prozent den Anforderungen hinsichtlich der Allgemeinbildung, die im Befehl vom 21. September 1961 formuliert worden waren. 1961 war dies nur bei 44 Prozent der angenommenen Offiziersbewerber der Fall gewesen ${ }^{126}$. Man stellte daher fest, die im Befehl enthaltenen Einschränkungen hinsichtlich der Allgemeinbildung könnten in Zukunft wegfallen ${ }^{127}$.

Dem trug die erneute Erhöhung der verbindlichen Einstellungsvoraussetzungen für Offiziersbewerber Rechnung, die das Politbüro im Rahmen der „Grundsätze für die Änderung der politischen und militärischen Erziehungs- und Bildungsarbeit an den Offiziersschulen der NVA“ am 3. Juli 1962 bestätigte ${ }^{128}$. Darin heißt es, die Veränderung der Gefechtsführung, die neue Ausrüstung und Bewaffnung der NVA und ihre zukünftigen Aufgaben verlangten die „allseitige Verbesserung der Aus-

122 Ebenda, Bl. 136.

123 Gesetz über die allgemeine Wehrpflicht. (Wehrpflichtgesetz). Vom 24. Januar 1962, GBl. I 1962, S. 2, 4.

124 Aus der Rede des Mitglieds des ZK der SED und Ministers für Nationale Verteidigung der DDR, Armeegeneral H. Hoffmann, zur Begründung des Wehrpflichtgesetzes vor der Volkskammer der DDR, 24. Januar 1961, in: Die Militär- und Sicherheitspolitik der SED, S. 297.

125 Einschätzung der Lage an den Offiziersschulen der NVA, 1962, SAPMO-BArch, DY 30/IV 2/12/32, Bl. 149.

126 Einschätzung der Werbung, Auswahl und Kommissionierung der Offiziersbewerber für das Ausbildungsjahr 1963. Protokoll des Kollegiums des Ministeriums für Nationale Verteidigung, 9. Juni 1962, BA-MA, AZN/28189, Bl. 79 f.

127 Ebenda, Bl. 84.

128 Protokoll Nr. 30/62 der Sitzung des Politbüros des ZK der SED, 3. Juli 1962, SAPMOBArch, DY 30/J IV 2/2/837, Bl. 8 . 
wahl der Offiziersbewerber"129. Die höheren Anforderungen, die im Zuge dieser Entwicklung an die Offiziere gestellt würden, machten es daher notwendig, daß die „Offiziersbewerber das Niveau der 10-Klassen allgemeinbildenden polytechnischen Oberschulen bzw. der erweiterten Oberschulen (Abitur) besitzen" müßten: „Deshalb sind ab 1964 die Offiziersbewerber aus den Absolventen dieser Schulen auszuwählen." 130 Unteroffiziere und Soldaten, die diese geforderte schulische Bildung nicht nachweisen konnten, hatten somit in der Regel keine Chance mehr, in die Offizierslaufbahn übernommen zu werden, selbst wenn sie über eine langjährige Truppenerfahrung verfügten.

Die SED setzte jetzt unverhohlen auf die Professionalisierung des Offiziersberufes. Wenn auch nicht in der Theorie, so entfernte man sich doch in der praktischen Kaderpolitik ab 1962 vom Bild des Offiziers als Vertreter der Arbeiterklasse in Uniform. Jetzt hieß es statt dessen, da die Offiziersausbildung die „Vorbereitung auf den Offiziers-Beruf“ sei, erscheine bei den Offiziersbewerbern eine "vorherige Ausbildung in einem anderen Beruf als nicht unbedingt erforderlich". Fehlende Erfahrungen in der sozialistischen Produktion glaubte man durch klassenmäßige Erziehung kompensieren zu können ${ }^{131}$. Die Werbung sollte daher vornehmlich unter den Absolventen weiterführender Schulen erfolgen, die unmittelbar „vor der Berufswahl" standen, wobei das Interesse vor allem auf Abiturienten gerichtet war ${ }^{132}$. Anscheinend versuchte die SED von nun an, den Offiziersberuf als eine ernstzunehmende Alternative zum Hochschulstudium und zu akademischen Berufen zu etablieren.

Es dürfte nicht zuletzt das Resultat dieser personalpolitischen Neuerungen gewesen sein, daß das Bildungsniveau der Offiziere bis Anfang 1963 spürbar anstieg. 17,7 Prozent der Offiziere verfügten jetzt über das Abitur und 31,4 Prozent über die Mittlere Reife. Nur noch 50,9 Prozent hatten allein eine Volksschulbildung vorzuweisen ${ }^{133}$. Obwohl Hoffmann auf der Kadertagung des Ministeriums für Nationale Verteidigung im Mai 1963 vermelden konnte, daß unterdessen 90 Prozent aller Offiziere der NVA eine abgeschlossene Offiziersausbildung besaßen und 80 Prozent der Regimentskommandeure über eine akademische Bildung verfügten ${ }^{134}$, war die Bestandssituation bei Offizieren mit einer spezifisch naturwissenschaftlich-technischen Qualifikation weiterhin prekär. So konnten bis Juni 1963 die 3994 vorhandenen Planstellen für Ingenieure und vergleichbare Verwendungen nur zu 47 Prozent besetzt werden; die 4700 Planstellen von Technikern sogar nur zu 25 Prozent ${ }^{135 .}$

129 Grundsätze für die Änderung der politischen und militärischen Erziehungs- und Bildungsarbeit an den Offiziersschulen der NVA, 1962, SAPMO-BArch, DY 30/IV 2/12/32, Bl. $126 \mathrm{ff}$.

130 Ebd., Bl. 129.

131 Einschätzung der Werbung, Auswahl und Kommissionierung der Offiziersbewerber für das Ausbildungsjahr 1963. Protokoll des Kollegiums des Ministeriums für Nationale Verteidigung, 9. Juni 1962, BA-MA, AZN/28189, Bl. 81.

132 Ebenda, Bl. 84.

133 Personalstatistik über die Zusammensetzung des Offiziersbestandes, 1. Januar 1963, BA-MA, VA-01/13877, Bl. 1.

134 Referat des Ministers für Nationale Verteidigung auf der Kadertagung des Ministeriums für Nationale Verteidigung, 24. Mai 1963, BA-MA, VA-01/13871, Bl. 8.

135 Probleme der Erziehung und Bildung in der NVA, die sich aus der modernen Militärtech- 
Die SED äußerte daher, der Bildungsstand der Kader auf mathematisch-naturwissenschaftlichem und militär-technischem Gebiet bleibe hinter den „objektiven Erfordernissen “ zurück ${ }^{136}$. Aus diesem Grund zielte man in den folgenden Jahren darauf ab, den Mangel an Fachleuten durch die „Werbung und Einstellung von Absolventen ziviler Hoch- und Fachschulen “ auszugleichen ${ }^{137 .}$

Ende 1963 wurde dann ein einheitliches System der Weiterbildung aller Offiziere eingeführt, um deren Ausbildung weiter zu verbessern ${ }^{138}$. Bereits am 13. Juni 1963 hatte der Nationale Verteidigungsrat in diesem Zusammenhang die Auflösung der vierzehn Offizierschulen der verschiedenen Waffengattungen und Teilstreitkräfte beschlossen. Vom Ausbildungsjahr 1964 an wurde die zentralisierte und vereinheitlichte Offiziersausbildung nur noch an den vier neugeschaffenen Offiziersschulen der Landstreitkräfte, Luftstreitkräfte/Luftverteidigung, Volksmarine und Grenztruppen durchgeführt. Alle Offiziersschüler erwarben jetzt neben ihrer militärischen Qualifikation auch ein Staatsexamen als zivilberuflichen Abschluß ${ }^{139}$. Mit der Umstrukturierung reagierte man auf die Erkenntnis, daß es an den Offiziersschulen bis dahin eine „zu starke Spezialisierung“ gegeben hatte ${ }^{140}$, weshalb beispielsweise die „allgemeine militärische Ausbildung der Spezialisten ... gegenüber ihren Spezialfächern vernachlässigt" worden war ${ }^{141}$.

Im Zuge dieser Veränderungen wurden die „allgemeinen Qualifikationsmerkmale“, denen die NVA-Offiziere zu entsprechen hatten, weiter verschärft. So mußte ein Offizier der Landstreitkräfte nunmehr als „Minimum“ die Kenntnisse der 10. Klasse der allgemeinbildenden polytechnischen Oberschule nachweisen können. Dieses Bildungsniveau reichte jedoch nicht für alle Dienststellungen aus; für einige war unterdessen ein Hochschulabschluß Voraussetzung. Offiziere, die ein Hochschulstudium etwa an der Militärakademie beginnen wollten, mußten die Kenntnisse der 12. Klasse der allgemeinbildenden Oberschule nachweisen können - zumindest in den Fächern Mathematik, Physik, Chemie und Russisch. Im Hinblick auf die Versetzung in höhere Dienststellungen waren jetzt aber nicht mehr nur schulische Bildung und fachliche Qualifikation entscheidend, sondern auch die Frage, inwieweit die Offiziere fundierte Erfahrungen in den jeweiligen Verwendungen im Truppen- oder Stabsdienst gesammelt hatten. In der Regel sollten Offiziere daher jeweils zwischen drei und vier Jahren in den einzelnen Dienststellungen eingesetzt worden sein, bevor sie erneut versetzt wurden ${ }^{142}$. Die SED war offensicht-

nik ergeben. Protokoll der 15. Sitzung des Nationalen Verteidigungsrates der DDR, 13. Juni 1963, BA-MA, DVW 1/39472, Bl. 49.

136 Probleme der Erziehung und Bildung in der NVA, die sich aus der modernen Militärtechnik ergeben, 7. Juni 1963, SAPMO-BArch, DY 30/IV A2/12/38, o. Pag.

137 Programm für die Mechanisierung und Automatisierung der Truppenführung in der NVA bis zum Jahre 1970. Protokoll der Sitzung der Leitung des Ministeriums für Nationale Verteidigung, 28. September 1964, BA-MA, AZN/28115, Bl. 73.

138 Zeittafel zur Militärgeschichte, S. 181.

139 Protokoll der 15. Sitzung des Nationalen Verteidigungsrates der DDR, 13. Juni 1963, BA-MA, DVW 1/39472, Bl. 6.

140 Einschätzung der Lage an den Offiziersschulen der NVA, 1962, SAPMO-BArch, DY 30/IV 2/12/32, Bl. 145.

141 Ebenda, Bl. 144.

142 Katalog über die Qualifizierungsmerkmale der Offiziersdienststellen der Landstreitkräfte, 1964, BA-MA, VA-01/13881, Bl. 9 f. 
lich bestrebt, eine gewisse Kontinuität in die Beförderungspraxis zu bringen, die es vor allem aufgrund des Personalmangels in den fünfziger Jahren nicht gegeben hatte.

Bis 1965 gelang es der Partei, das Bildungs- und Ausbildungsniveau noch einmal entscheidend zu erhöhen, ohne letztlich jedoch den gewünschten Standard zu erreichen. Am Jahresende verfügten 19,0 Prozent aller Offiziere über das Abitur und 39,3 Prozent über die Mittlere Reife. Mit 41,7 Prozent war der Anteil der Offiziere mit Volksschulbildung nunmehr deutlich gesunken ${ }^{143}$. Hinsichtlich der fachlichen Qualifikation konnten bereits 10,4 Prozent der NVA-Offiziere eine akademische Bildung vorweisen, die sie an einer zivilen oder militärischen Hochschule erworben hatten. 0,8 Prozent hatten Kurse an Militärakademien besucht. 72,7 Prozent der Offiziere besaßen eine zivile oder militärische Fachschulausbildung. Dazu zählten auch diejenigen Offiziere, die das dritte oder vierte Jahr der Ausbildung an einer Offiziersschule absolviert hatten. 3,3 Prozent verfügten über eine zweijährige und 5,4 Prozent über eine einjährige Ausbildung an einer Offiziersschule. 2,1 Prozent der Offiziere hatten nur einen Kurzlehrgang und 2,2 Prozent ausschließlich eine zivile Parteischule absolviert. 3,5 Prozent der Offiziere hatten noch immer keine militärische Schule besucht ${ }^{144}$.

Ein weiteres Kennzeichen für die Professionalisierung der NVA war der Versuch der SED-Führung, auch bei der Auswahl und Ausbildung der Politoffiziere ein höheres Niveau zu erreichen ${ }^{145}$. Am 23. Juni 1961 beschloß der Nationale Verteidigungsrat daher, die politische Bildung aller Offiziersbewerber zu verbessern, um im Anschluß an die allgemeine Offiziersausbildung aus diesem Kreis einen qualifizierteren Nachwuchs an Politoffizieren gewinnen zu können. Dies stellte eine konsequente Weiterentwicklung der Neuerungen des Vorjahres dar. Denn bereits 1960 war die grundsätzliche Eigenständigkeit der Politoffizierslaufbahn aufgegeben worden. Politoffiziere wurden seitdem aus den Reihen der politisch aktivsten Fachoffiziere gewonnen ${ }^{146}$. Vom 1. Januar 1961 an sollten nunmehr allen Offiziersschülern „als Minimum in der politischen Ausbildung die Kenntnisse des Programms einer Bezirksparteischule" vermittelt werden ${ }^{147}$.

Der SED ging es aber auch darum, die politische, militärische und militär-technische Aus- und Weiterbildung der Politoffiziere erheblich zu verbessern. Diese sollte systematisiert werden und auf einem höheren Niveau erfolgen. So waren die Politoffiziere der Bataillone nunmehr auch in den Qualifizierungslehrgängen für Bataillonskommandeure auszubilden. Die zukünftigen Politoffiziere wurden dabei im Rahmen einer speziellen Ergänzungsausbildung auf ihren „vorgesehenen Einsatz“ vorbereitet. Auf der nächsthöheren Ebene sollten die Politoffiziere der Regimenter an der Militärakademie „Friedrich Engels“ ausgebildet werden. Als politische $\mathrm{Zu}$ satzqualifikation erhielten sie „als Minimum Kenntnisse des Ein-Jahreslehrganges

143 Kaderjahrbuch 1965, BA-MA, VA-01/32444, Bl. 272.

144 Ebenda, Bl. 177.

145 Vgl. Fingerle, Waffen in Arbeiterhand?, S. 124.

146 Vgl. Kap. III.3. dieser Studie.

147 Plan zur Heranbildung politisch und militärisch hochqualifizierter Offiziere und zur Sicherung des Nachwuchses sowie der Qualifizierung von Partei- und Politkadern der NVA. Protokoll der 6. Sitzung des Nationalen Verteidigungsrates der DDR, 23. Juni 1961, BA-MA, DVW 1/39463, Bl. 95. 
der Parteihochschule ,Karl-Marx““. Die „höheren politisch-militärischen Kader“ sollten in einem dreijährigen Studium am neuzuschaffenden „Institut für Gesellschaftswissenschaften der Militärakademie" ausgebildet werden ${ }^{148}$. Durch dieses verbesserte Ausbildungssystem hoffte man, „erfahrene Truppenkommandeure als Offiziere für politische Arbeit erhalten“ zu können, die „größere Lebenserfahrung und hohe militärische Qualitäten besitzen" würden ${ }^{149}$. Diese Veränderungen machten es schließlich möglich, die Politschule der NVA, an der bis dahin auch die politische Ausbildung der zukünftigen Politoffiziere stattgefunden hatte, zum 31. Dezember 1961 aufzulösen ${ }^{150}$. In Anbetracht der „zahlenmäßigen Begrenzung“ der Armee erachtete die Leitung des Ministeriums für Nationale Verteidigung die Einrichtung einer Politoffiziersschule als "nicht zweckmäßig"151.

1963 wurden die speziellen Lehrgänge für die Politoffiziere der Bataillone jedoch offenbar eingestellt, so daß von da an „kein System der Heran- und Weiterbildung von Politoffizieren mehr" bestand, wie die Verwaltung Kader rückblickend feststellte. Anscheinend stieß das SED-Konzept vom Offizier als einem universell einsetzbaren und politisch wie militärisch qualifizierten Vorgesetzten jedoch bald an seine Grenzen. Zumindest stellten die Politische Hauptverwaltung und die ZK-Abteilung für Sicherheitsfragen 1964/1965 bei Kontrollen wiederholt fest, daß eine „Vielzahl von Politoffizieren ... keine ihrer Dienststellung entsprechende und für die politische Arbeit notwendige militärische Ausbildung" besaß. Anscheinend erwies es sich in der Praxis als nicht möglich, Politoffiziere auszubilden, die ohne weiteres auch in der militärischen Truppenführung einzusetzen waren. Insofern war es konsequent, wenn die Partei auf diesen Zustand mit einer wieder stärkeren Spezialisierung der Politoffiziersausbildung reagierte. 1965 wurden daher die Rekrutierungsrichtlinien neu fixiert. Die Auswahl der zukünftigen Politoffiziere sollte jetzt bereits frühzeitig an den Offiziersschulen erfolgen. Im Verlauf der Ausbildung galt es anhand der vorhandenen „Fähigkeiten und Neigungen der Offiziersschüler“ festzustellen, für welche von ihnen ein späterer Einsatz als Politoffizier „zweckmäBig“ erschien. Diese Offiziersschüler sollten dann durch „geeignete Maßnahmen gefördert werden", um sie auf ihre zukünftige Verwendung vorzubereiten ${ }^{152}$.

Kennzeichnend für die weitere Entwicklung der DDR-Streitkräfte nach dem Mauerbau war insbesondere ihre umfassende Modernisierung. Die SED bemühte sich dabei zum einen, die militärischen Führungsprozesse auf der Grundlage der

148 Ebenda, Bl. 95 f.

149 Konzeption zur Heranbildung politisch und militärisch hochqualifizierter Offiziere und zur Sicherung des Nachwuchses sowie der Qualifizierung von Politoffizieren. Protokoll der Sitzung der Leitung des Ministeriums für Nationale Verteidigung, 9. Juni 1961, BA-MA, AZN/28076, Bl. 102.

150 Auflösung der Politschule der NVA. Befehl Nr. 50/61 des Ministers für Nationale Verteidigung, 9. August 1961, BA-MA, VA-01/5903, Bl. 117.

151 Nach eigenen Angaben war die NVA dadurch die einzige sozialistische Armee, die nicht über spezielle Institutionen für die Ausbildung von Politoffizieren verfügte. Konzeption zur Heranbildung politisch und militärisch hochqualifizierter Offiziere und zur Sicherung des Nachwuchses sowie der Qualifizierung von Politoffizieren. Protokoll der Sitzung der Leitung des Ministeriums für Nationale Verteidigung, 9. Juni 1961, BA-MA, AZN/28076, Bl. 102.

152 Teilbeitrag der Verwaltung Kader zur Einarbeitung in die Gesamtanalyse und Grundkonzeption der Entwicklung der NVA, 10. März 1967, BA-MA, VA-01/5679, Bl. $87 \mathrm{ff}$. 
neuesten wissenschaftlichen Erkenntnisse zu reorganisieren und $\mathrm{zu}$ verbessern. Zum anderen kam es im Zuge der Ausstattung mit neuen Waffensystemen zu einer verstärkten Technisierung und Spezialisierung der NVA. Ulbricht teilte Chruschtschow in diesem Zusammenhang im Januar 1962 mit: „In Durchführung der Empfehlungen über die einheitliche Ausstattung der Streitkräfte der Teilnehmerstaaten des Warschauer Vertrages werden in der Nationalen Volksarmee ... moderne Kampftechnik und Bewaffnung, die bisher noch nicht vorhanden waren, eingeführt. Sämtliche Waffen und Geräte werden aus der UdSSR importiert. "153

Im Rahmen der Umrüstung wurden 1962 unter anderem in den Landstreitkräften die neuen Waffengattungen „Truppenluftabwehr" und „Raketentruppen und Artillerie" geschaffen. Letztere erhielt sowohl taktische als auch operativ-taktische Boden-Boden-Raketen. Die NVA verfügte nunmehr über atomare Trägersysteme. Auch gehörte etwa der mittlere Kampfpanzer T-54 Mitte der sechziger Jahre zur Standardausrüstung der gepanzerten Verbände. Zu diesem Zeitpunkt begann man zudem mit der Einführung des mittleren Kampfpanzers T-55. Darüber hinaus wurden die motorisierten Schützeneinheiten jetzt mit Panzerabwehrlenkraketen ausgestattet ${ }^{154}$. Die Modernisierung der Luftstreitkräfte/Luftverteidigung war vor allem durch die Ausrüstung mit leistungsfähigeren MIG-21 Jagdflugzeugen bestimmt, die es der NVA ermöglichten, ihre Aufgaben im einheitlichen Luftverteidigungssystem des Warschauer Paktes zu erfüllen. Darüber hinaus wurde diese Teilstreitkraft auch mit Fla-Raketensystemen und neuen Mehrzweckkampfhubschraubern ausgerüstet. Die Seestreitkräfte erhielten seit 1962 Raketenschnellboote und begannen eigene Landungskapazitäten aufzubauen ${ }^{155}$.

Im Hinblick auf die Modernisierung der militärischen Führungsprozesse in der NVA ging ein entscheidender Impuls vom XXII. Parteitag der KPdSU im Oktober 1961 aus. Die sowjetische Führung verfolgte allgemein die Absicht, mittels neuer Technologien wie der elektronischen Datenverarbeitung oder der verstärkten Automatisierung die Effizienz der staatlichen und ökonomischen Planungs- und Leitungssysteme sowie die allgemeine Arbeitsproduktivität zu steigern. Die von der KPdSU zu diesem Zweck propagierten Reformkonzepte der „wissenschaftlichtechnischen Revolution" und der "Produktivkraft Wissenschaft" wurden von der SED im Anschluß mit gewisser zeitlicher Verzögerung auf dem VI. Parteitag im Januar 1963 rezipiert. Dies geschah im Rahmen des „Neuen Ökonomischen Systems der Planung und Leitung" der Volkswirtschaft (NÖSPL), mit dessen Hilfe die SED nach der Abriegelung der DDR nunmehr den „umfassenden Aufbau des Sozialismus" verwirklichen wollte. Das NÖSPL zielte dabei nicht zuletzt auf eine Verwissenschaftlichung der staatlichen und wirtschaftlichen Leitungsprozesse ab. Auf der Grundlage des „höchsten Standes von Wissenschaft und Technik“ sollte unter anderem eine Versachlichung der Leitungstätigkeit erzielt werden. Dabei wollte die SED eine beschränkte Selbststeuerung der jeweiligen Teilbereiche zulassen, ohne jedoch die übergeordnete Kontrollkompetenz der Partei zu reduzieren ${ }^{156}$.

153 Ulbricht an Chruschtschow, 31. Januar 1962, SAPMO-BArch, DY 30/J IV 2/202/245, o. Pag.

154 Höhn, Einige Entwicklungsprobleme der Landstreitkräfte der NVA, S. 419 f.

155 Wenzke, Die Nationale Volksarmee, S. $446 \mathrm{ff}$.

$156 \mathrm{Vgl}$. Meuschel, Legitimation und Parteiherrschaft in der DDR, S. $183 \mathrm{ff}$. 
Diesen Reformansatz übertrug die SED umgehend auch auf die Streitkräfte. Die bestehenden Defizite in den militärischen Führungsprozessen sowohl hinsichtlich des Grades der Technisierung als auch in bezug auf ihre Effizienz und Leistungsfähigkeit dürften der Parteiführung und der Leitung des Ministeriums für Nationale Verteidigung schon seit längerem hinlänglich bekannt gewesen sein ${ }^{157}$. Unter den veränderten politischen Bedingungen wurden sie jetzt aber offen thematisiert: So äußerte man, daß sich das System sowie die Formen und Methoden der Truppenführung im wesentlichen auf dem Stand „der letzten Jahre des 2. Weltkrieges“ befänden. Die Strukturen der Stäbe seien „zu aufgebläht und unbeweglich“, und die technische Ausstattung der Führungseinrichtungen entspreche im allgemeinen nicht den Anforderungen, die im Hinblick auf militärische Operationen im Rahmen der Ersten Strategischen Staffel erfüllt werden müßten ${ }^{158}$. Interessanterweise wurde dabei nicht nur auf „Rückstände“ gegenüber den sowjetischen Streitkräften, sondern auch gegenüber der „US-Armee“ verwiesen. Zudem monierte man das späte zeitliche „Aufgreifen der Problematik“159.

Hoffmann stellte daher im Oktober 1963 grundsätzlich fest: „Der sich immer stärker abzeichnende Widerspruch zwischen der Ausrüstung der Truppen mit modernster Kampftechnik ... und den derzeitigen Führungsmethoden und -möglichkeiten der Kommandeure und Stäbe kann nur durch den Einsatz modernster Führungsmittel, verbunden mit der allseitigen Vervollkommnung der Arbeitsmethoden der Kommandeure und Stäbe, der Struktur der Führungsorgane und der Organisation der Führungsstellen sowie der Erhöhung des operativ-taktischen und militärtechnischen Ausbildungsstandes der Offizierskader gelöst werden." Daher sei die „Mechanisierung und Automatisierung der Truppenführung ... eine der wichtigsten Aufgaben“ der nächsten Jahre. Durch die Anordnung vom 24. Oktober 1963 leitete der Minister erste Maßnahmen zu deren Lösung ein ${ }^{160}$.

In der Folgezeit wurde ein Arbeitsprogramm zur „Ausarbeitung und Durchsetzung neuer Formen und Methoden in der Führungs- und Leitungstätigkeit der $\mathrm{Na}$ tionalen Volksarmee und der Anwendung modernster technischer Führungsmittel“ entworfen. In diesem Zusammenhang bezeichnete man die ZK-Beschlüsse zu den „Fragen des neuen ökonomischen Systems der Planung und Leitung der Volkswirtschaft" ausdrücklich als ein „Programm für die schöpferische Lösung der herangereiften Fragen der Truppenführung “ in den Streitkräften. Zunächst sollte eine wissenschaftliche Analyse des "gesamten Führungsprozesses“ in der NVA vorgenommen werden. Auf deren Basis beabsichtigte man unter Berücksichtung der „neuen Auffassungen über die moderne Truppenführung“ die „Erarbeitung und schrittweise Einführung eines komplexen automatisierenden Führungssystems“. Die

157 Vgl. Bericht der Brigade des ZK der SED in der 11. Mot. Schützendivision der NVA, 1961, SAPMO-BArch, DY 30/IV 2/12/24, Bl. $45 \mathrm{ff}$.

158 Entwurf eines Arbeitsprogrammes zur Ausarbeitung und Durchsetzung neuer Formen und Methoden in der Führungs- und Leitungstätigkeit der NVA und der Anwendung modernster technischer Führungsmittel, 1964, SAPMO-BArch, DY 30/IV A2/12/49, o. Pag.

159 Programm für die Mechanisierung und Automatisierung der Truppenführung in der NVA bis zum Jahre 1970. Protokoll der Sitzung der Leitung des Ministeriums für Nationale Verteidigung, 28. September 1964, BA-MA, AZN/28115, Bl. 16.

160 Aufgaben auf dem Gebiet der Mechanisierung und Automatisierung der Truppenführung in der NVA. Anordnung Nr. 35/63 des Ministers für Nationale Verteidigung, 24. Oktober 1963, BA-MA, VA-01/5614, Bl. 1 ff. 
technischen Grundlagen hierfür sollten Kybernetik, Funkelektronik und elektronische Rechenautomatik bilden. Auch wollte man eine "weitgehende Automatisierung und Mechanisierung der gesamten Stabsarbeit“ mit den Mitteln „Kleinstautomatisierung und Mechanisierung " sowie der "modernen Bürotechnik“ erreichen. Schließlich sollten diese Neuerungen ihren Niederschlag auch in veränderten Führungsstrukturen finden ${ }^{161}$.

Der Nationale Verteidigungsrat bestätigte dann am 8. Januar 1965 das „Programm für die Mechanisierung und Automatisierung der Truppenführung in der Nationalen Volksarmee bis zum Jahre 1970“. Die Ausrüstung der NVA mit den neuen technischen Führungsmitteln sollte beschleunigt werden, um die Mechanisierung der Truppenführung bereits 1967/1968 sicherstellen zu können. In bezug auf die Automatisierung war es das Ziel, 1970 ,im wesentlichen“ den Stand der Sowjetarmee zu erreichen. Daß dies in Abstimmung mit dem Vereinten Kommando des Warschauer Paktes geschehen sollte, hatte offensichtlich mit der Absicht zu tun, die Kompatibilität der Prinzipien, Mittel und Strukturen der militärischen Truppenführung innerhalb des Bündnisses zu optimieren ${ }^{162}$. Ferner befürwortete der Nationale Verteidigungsrat, Vereinbarungen mit der UdSSR über die Qualifizierung von NVA-Offizieren an den sowjetischen Militärakademien und zivilen Bildungseinrichtungen auf dem Gebiet der Mechanisierung und Automatisierung zu treffen. Darüber hinaus wurde beschlossen, diese Ausbildungsinhalte und „umfangreiche naturwissenschaftlich-technische Kenntnisse" auch in die zu überarbeitenden Ausbildungsprogramme der Offiziersschulen und der Militärakademie der NVA aufzunehmen ${ }^{163}$. Die herausragende Bedeutung, die der Modernisierung der militärischen Führungsprozesse durch die SED mittlerweile beigemessen wurde, kam nicht zuletzt darin zum Ausdruck, daß der Minister für Nationale Verteidigung im Mai 1965 befahl, die Dienststellung eines Stellvertreters „für Mechanisierung und Automatisierung der Truppenführung" zu schaffen ${ }^{164}$.

Im Zuge der zunehmenden Modernisierung und Professionalisierung der NVA $^{165}$ reduzierte sich auch die direkte Einflußnahme der Parteiorganisationen und der Politorgane auf die spezifisch militärischen Entscheidungsprozesse. Eine Ursache für diese Entwicklung war die veränderte Arbeitsweise des Parteiapparates selbst, die die SED nach dem Mauerbau in Gang gesetzt hatte. Die Partei dürfte zu

161 Entwurf eines Arbeitsprogrammes zur Ausarbeitung und Durchsetzung neuer Formen und Methoden in der Führungs- und Leitungstätigkeit der NVA und der Anwendung modernster technischer Führungsmittel, 1964, SAPMO-BArch, DY 30/IV A2/12/49, o. Pag. Bis zu diesem Zeitpunkt etwa war der Personalumfang der Stäbe in der NVA im Vergleich zu den sowjetischen Streitkräfte noch um durchschnittlich 28 Prozent größer. Programm für die Mechanisierung und Automatisierung der Truppenführung in der NVA bis zum Jahre 1970. Protokoll der Sitzung der Leitung des Ministeriums für Nationale Verteidigung, 28. September 1964, BA-MA, AZN/28115, Bl. 20.

162 Programm für die Mechanisierung und Automatisierung der Truppenführung in der NVA bis zum Jahre 1970. Protokoll der 21. Sitzung des Nationalen Verteidigungsrates der DDR, 8. Januar 1965, BA-MA, DVW 1/39478, Bl. 29.

163 Ebenda, Bl. 40.

164 Über die weiteren Aufgaben zur Mechanisierung und Automatisierung der Truppenführung in der NVA. Befehl Nr.22/65 des Ministers für Nationale Verteidigung, 30. April 1965, BA-MA, VA-01/12918, Bl. 63.

165 Vgl. Johnson, Die Streitkräfte des Warschauer Paktes in Mitteleuropa, S. $123 \mathrm{ff}$. 
der Erkenntnis gelangt sein, daß sich eine anhaltend extensive Politisierung militärischer Diskussions- und Entscheidungsprozesse als ineffizient erweisen und im Hinblick auf die Erhöhung der Einsatzbereitschaft eine weitgehend kontraproduktive Wirkung entfalten würde. Ziel war es daher, die Einflußnahme der Partei auf die militärischen Führungsprozesse zu modifizieren. Einerseits sollten die Kontrollfunktion des Parteiapparates gestärkt werden und die direkten Interventionen demgegenüber an Bedeutung verlieren ${ }^{166}$. Andererseits strebte man die Versachlichung der Parteiarbeit an. So befand Hoffmann, die SED trete in der Periode des umfassenden Aufbaus des Sozialismus in eine neue, höhere Stufe ihrer Entwicklung ein. Die Parteiorganisationen in der NVA müßten dem durch „eine wirklich sach- und fachkundige Parteiarbeit" Rechnung tragen ${ }^{167}$. Diese Forderung präzisierte der Chef der Politischen Hauptverwaltung Verner Anfang 1964. Er sagte, die Parteiarbeit und die gesamte politische Arbeit seien näher an die militärischen Aufgaben heranzubringen. Die Politorgane und Parteiorganisationen müßten "noch tiefer in die Probleme der Gefechtsbereitschaft" eindringen und die „inneren Zusammenhänge“ beachten: „Dann werden sie weitaus sachkundiger auf die Erfüllung aller Aufgaben der Gefechtsausbildung Einfluß nehmen und rechtzeitig Maßnahmen zur Beseitigung von Mängeln ... ergreifen können." 168

Damit beschleunigte die SED jedoch den Prozeß der Autonomisierung der Truppenführung, der sich im Verlauf der Professionalisierung der NVA ohnehin immer deutlicher abzeichnete. Spätestens 1965 mußte die SED-Führung daher feststellen, daß sich die Rolle des Parteiapparates in den Streitkräften in einem Maße veränderte, das über das ursprünglich angestrebte Ziel hinausging. Hoffmann sah sich daher zu der Forderung gezwungen, es solle „endgültig von der falschen Praxis abgegangen“" werden, „unter der Devise ,Versachlichung der Parteiarbeit" Leitungssitzungen und Versammlungen so durchzuführen, daß sie sich in ihrem Inhalt nicht von Kommandeursbesprechungen und Dienstversammlungen " unterschieden ${ }^{169}$. In diesem Zusammenhang kritisierte man auch, daß die "politisch-ideologische Seite der Arbeit der Grundorganisation als ihre eigentliche Aufgabe zur maximalen Erfüllung der militärischen Aufgaben in den Hintergrund gedrängt und vernachlässigt" werde ${ }^{170}$. Je mehr aber an die Einsatzbereitschaft der NVA auch von Seiten der Parteiführung in erster Linie spezifisch militärische Bewertungsmaßstäbe angelegt wurden, desto mehr verlor eine rein politische Argumentationsweise in der Führungstätigkeit an Bedeutung. Auf diese Weise verringerte sich zugleich der Spielraum der Parteiorganisationen und Politorgane, auf die militärischen Diskussionsund Entscheidungsprozesse nachhaltig Einfluß zu nehmen. Ihre politischen Argumentationsmuster verfügten offenkundig kaum mehr über Anschlußfähigkeit an die militärischen Problemstellungen. Vor allem dürfte der Mangel an Problemlösungskompetenz ein entscheidender Grund dafür gewesen sein, weshalb der Parteiappa-

166 Vgl. Kap. VIII.2. dieser Studie.

167 Aus dem Referat des Mitglieds des ZK der SED und Ministers für Nationale Verteidigung der DDR, Armeegeneral H. Hoffmann, auf der Kommandeurstagung der NVA, 11. November 1963, in: Die Militär- und Sicherheitspolitik der SED, S. 315.

168 Verner, Für die weitere Stärkung der führenden Rolle der Partei, S. 167.

169 Hoffmann, In den Parteiorganisationen, S. 4.

170 Raubach, Das Ausbildungsjahr 1964/65 und die höheren Forderungen, S. 32. 
rat sowohl im Rahmen der militärischen Führungsarbeit als auch im Truppenalltag selbst immer häufiger übergangen wurde ${ }^{171}$.

Insofern war es symptomatisch, wenn im Anschluß an eine Übung der 11. Mot. Schützendivision im Frühjahr 1962 festgestellt wurde: „Die Politabteilung war kaum an einer Entschlußvorbereitung beteiligt, der Einfluß des Leiters der Politabteilung auf die Entschlußfassung fehlte völlig ...."172 Die reduzierte Bedeutung des Parteiapparates zeigte sich beispielsweise auch darin, daß in der 9. Panzerdivision im ersten Halbjahr 1963 gegen 229 Mitglieder und Kandidaten der SED eine Disziplinarstrafe verhängt wurde, man jedoch nur gegen 11 von ihnen gleichzeitig Parteierziehungsmaßnahmen einleitete ${ }^{173}$. Zudem scheint immer häufiger die Neigung bestanden zu haben, Parteiversammlungen zu verschieben oder ganz ausfallen zu lassen. So kritisierte Verner im Dezember 1962, daß etwa im August des Jahres in 202 Grundorganisationen der SED in der NVA und im Oktober in 184 Grundorganisationen keine Parteiversammlungen stattgefunden hätten ${ }^{174}$. Darüber hinaus war es üblich geworden, daß leitende Offiziere nicht mehr regelmäßig an Parteiversammlungen teilnahmen ${ }^{175}$. Dies geschah nicht selten mit dem Hinweis auf die starke zeitliche Inanspruchnahme durch die militärische Führungstätigkeit. Im übrigen war es selten, daß man leitende Offiziere oder Kommandeure auf Parteiversammlungen im Zusammenhang mit ihrer dienstlichen Tätigkeit offen kritisierte ${ }^{176}$. Während der Überprüfung der Führungstätigkeit in der 9. Panzerdivision im Dezember 1963 etwa wurden Zweifel geäußert, ob „die leitenden Offiziere wegen mangelnder Erfüllung ihrer funktionellen Pflichten überhaupt kritisiert" werden könnten. Ein Parteisekretär sagte sogar: „Wir hatten Angst, Autoritäten zu kritisieren."177 Diese Äußerungen verdeutlichen, daß die SED faktisch mit ihrem Anspruch gescheitert war, alle Parteimitglieder und damit auch die Vertreter der militärischen Truppenführung sollten gleichermaßen der einheitlichen Parteidisziplin unterliegen. Es erwies sich letztlich als Fiktion, die militärischen Vorgesetztenverhältnisse könnten in den Parteiorganisationen keine Rolle spielen. Eine Trennung beider Bereiche ließ sich in der Praxis kaum aufrechterhalten, da es sich, wenn auch in verschiedenen Funktionen, jeweils um dieselben Personen handelte ${ }^{178}$.

Die Autonomisierung der militärischen Truppenführung war neben der Modernisierung der NVA eine entscheidende Voraussetzung für die zunehmende Leistungssteigerung der DDR-Streitkräfte in der Phase nach dem Mauerbau. Angesichts der 1961 beginnenden Eingliederung der NVA in die Erste Strategische Staffel

171 Vgl. Kap. IX. dieser Studie.

172 Information über einige Probleme im Verlauf der Übung bei der 11. MSD, 1962, SAPMOBArch, DY 30/IV 2/12/38, Bl. 170.

173 Bericht über die Ergebnisse der Überprüfung der Führungstätigkeit in der 9. Panzerdivision, 2. Dezember 1963, BA-MA, VA-01/12945, Bl. 13.

174 Protokoll der 2. Tagung der IV. Delegiertenkonferenz der Parteiorganisationen der SED in der NVA, 1./2. Dezember 1962, BA-MA, VA-P-01/559, Bl. 62.

175 Protokoll der Parteiaktivtagung der SED in der NVA, 26. März 1962, BA-MA, VA-P-01/555, Bl. 35.

176 Vgl. Stellungnahme zur Jahresanalyse der Parteikontrollkommission bei der Politischen Hauptverwaltung der NVA, 19. Januar 1965, SAPMO-BArch, DY 30/IV A2/12/35, o. Pag.

177 Bericht über die Ergebnisse der Überprüfung der Führungstätigkeit in der 9. Panzerdivision, 2. Dezember 1963, BA-MA, VA-01/12945, Bl. 17.

178 Vgl. Lolland, Zu Befehl, Genosse Unterleutnant, S. $88 \mathrm{ff}$. 
des Warschauer Paktes war ein Verbesserung der militärischen Leistungsfähigkeit unerläßlich. Vor allem von sowjetischer Seite dürfte in diesem Zusammenhang massiv darauf gedrungen worden sein, schnell substantielle Veränderungen zu erzielen. Das war aber nicht zuletzt wegen der umfassenden Umrüstungsmaßnahmen in allen Teilstreitkräften kurzfristig nur bedingt möglich. So stellte Hoffmann im März 1962 fest, eine kurz zuvor vom Ministerium für Nationale Verteidigung zusammen mit Vertretern des Vereinten Kommandos durchgeführte Inspektion zweier Verbände der Landstreitkräfte und der Luftstreitkräfte/Luftverteidigung habe „in der Gesamteinschätzung der einzelnen Truppenteile und Einheiten ... in der Regel genügende und befriedigende, manchmal auch gute Ergebnisse" erbracht. Bei der Herstellung der Gefechtsbereitschaft hätten sich jedoch noch genügend Mängel gezeigt $^{179}$. Anläßlich der IV. Delegiertenkonferenz der Parteiorganisationen der SED in der NVA Ende Juni 1962 zog der damalige Chef der Verwaltung Ausbildung, Generalleutnant Kurt Wagner, insgesamt aber eine positive Bilanz: „Die Erfolge, die wir in der Gefechtsausbildung im 1. Halbjahr 1962 zu verzeichnen haben, sind ohne Zweifel im Verhältnis zum Stand der Gefechtsbereitschaft des Jahres 1961 große Schritte nach vorn gewesen. " 180

Großen Einfluß auf die Steigerung der Leistungsfähigkeit der NVA hatte die Anfang 1962 eingeführte allgemeine Wehrpflicht. Besonders positiv wirkte sich das durchschnittlich höhere Bildungsniveau und die bessere Motivation der als Mannschaftsdienstgrade dienenden Wehrpflichtigen aus. Diesbezüglich äußerte beispielweise ein Oberstleutnant des MSR 23 Mitte des Jahres zufrieden: „Die erste Erfahrung ist die, daß die Erwartungen, die wir in unsere Wehrpflichtigen gesetzt hatten, weit in positiver Hinsicht übertroffen wurden. Wir haben Soldaten bekommen, die eine große Bereitwilligkeit, Aufgeschlossenheit und einen fleißigen Lerneifer zeigen." 181

Insgesamt gelang es, die militärische Einsatzbereitschaft in den ersten zwei Jahren nach dem Mauerbau so zu steigern, daß die NVA bereits Ende 1963 dazu in der Lage war, im Rahmen von Großverbänden der Vereinten Streitkräfte des Warschauer Paktes zu operieren. Das Manöver „Quartett“ im Oktober des Jahres zeigte die Befähigung der DDR-Streitkräfte, nunmehr auf Arme-Ebene mit den sowjetischen, tschechoslowakischen und polnischen Streitkräften zusammenwirken zu können. Zudem wurde der Nachweis erbracht, daß die militärische Truppenführung der NVA ihre operativen Fähigkeiten weiter optimiert hatte. So übernahm der Minister für Nationale Verteidigung die Leitung des Manövers, und hohe NVAGenerale kommandierten als Befehlshaber integrierte Armeen der Vereinten Streitkräfte182. In der anschließenden Bewertung hieß es, die beteiligten Stäbe, Verbände und Truppenteile der NVA hätten „bis zum Beginn der Übung einen bisher uner-

179 Protokoll der Parteiaktivtagung der SED in der NVA, 26. März 1962, BA-MA, VA-P-01/555, Bl. 64.

180 Protokoll der IV. Delegiertenkonferenz der Parteiorganisationen der SED in der NVA, 29. Juni-1. Juli 1962, BA-MA, VA-P-01/558, B1. 271.

181 Ebenda, Bl. 224. Auch die DDR-Streitkräfte profitierten somit von den charakteristischen Vorzügen, die eine Wehrpflichtarmee gegenüber allein aus Freiwilligen bestehenden Streitkräften besitzt.

182 Vgl. Kap. VII.1. dieser Studie. 
reichten Stand der Gefechtsbereitschaft" erreicht ${ }^{183}$. Außerdem bezeichnete man es als bemerkenswert, daß die NVA-Einheiten bei den Manöveroperationen „den verbündeten Truppen nicht nachstanden "184.

Das Politbüro stellte Ende 1963 fest, die weitere „Erhöhung der Gefechtsbereitschaft und der Kampfkraft der Truppenteile und Verbände" sei einer der bedeutenden Erfolge, den die NVA im abgelaufenen Ausbildungsjahr erzielt habe. Vor allem die großen Übungen im Bündnisrahmen hätten den "hohen operativ-taktischen Ausbildungsstand der Kommandeure, Stäbe und der Truppen" bewiesen. Als Fazit der bisherigen militärpolitischen Anstrengungen äußerte man: „Die Nationale Volksarmee ist in der Lage, gemeinsam mit der ruhmreichen Sowjetarmee und den anderen sozialistischen Bruderarmeen die ihr vom Vereinten Oberkommando übertragenen Aufgaben zu lösen und erfolgreiche Kampfhandlungen unter beliebigen Bedingungen der Lage, mit und ohne Einsatz von Kernwaffen zu führen. " 185

Vor allem wurden im Hinblick auf die verbesserten Führungsqualitäten der Offiziere mehr und mehr die Erfolge der versachlichten Personalpolitik sichtbar. Dies zeigte beispielsweise die Überprüfung der 1. Mot. Schützendivision im August 1964. Im Untersuchungsbericht heißt es, die Regimentskommandeure hätten ihre gewachsene Fähigkeit unter Beweis gestellt, ,auch ohne auf Weisungen übergeordneter militärischer Organe zu warten, selbständig und schnell zu reagieren“. Als einen entscheidenden Grund hierfür wertete man die „wachsende Zahl akademisch gebildeter Offiziere" ${ }^{186}$. Anscheinend bildete das Offizierskorps im Zuge der Professionalisierung der NVA ein zunehmend eigenverantwortliches, zielorientiertes und fachbezogenes Führungsverhalten aus.

Darüber hinaus verbesserten sich offenbar auch die Fähigkeiten der NVA-Teilstreitkräfte, gemeinsame militärische Operationen durchzuführen. Dies zeigte unter anderem eine Flottenübung, die im September 1964 stattfand. Im Ergebnis stellte man fest, die Übung habe bewiesen, daß die Einheiten und Verbände der Volksmarine in der Lage seien, ,im Zusammenwirken mit den Land- und Luftstreitkräften/ Luftverteidigung ... erfolgreich taktische Seelandungen durchzuführen" 187 . Gerade das mangelhafte Zusammenwirken der Teilstreitkräfte war in den fünfziger Jahren eines der Hauptprobleme bei der Steigerung der Einsatzbereitschaft der NVA gewesen $^{188}$.

Am Ende des Ausbildungsjahres 1964/1965 gelangte die Leitung des Ministeriums für Nationale Verteidigung schließlich zu dem Schluß, die Resultate der Übungen und Überprüfungen gestatteten die „Einschätzung, daß die Stäbe, Verbände und Truppenteile aller Teile der Nationalen Volksarmee gefechtsbereit“ seien. „Mit

183 Bericht über die in der Zeit vom 11. bis 15. September 1963 durchgeführte Übung „Quartett“, 18. Januar 1964, BA-MA, VA-01/12946, Bl. 104.

184 Bericht über die in der Zeit vom 11. bis 15. September 1963 durchgeführte Übung „Quartett“, 18. Januar 1964, BA-MA, VA-01/5199, Bl. 23.

185 Grundsätze der politischen und militärischen Arbeit der NVA im Ausbildungsjahr 1963/1964. Arbeitsprotokoll Nr. 39/63 der Sitzung des Politbüros des ZK der SED, 5. November 1963, SAPMO-BArch, DY 30/J IV 2/2A/997, o. Pag.

186 Bericht über die Untersuchung des Standes der politisch-ideologischen Arbeit in der 1. Mot. Schützendivision, 12. August 1964, BA-MA, VA-01/13870, Bl. 101.

187 Information über die vom 7. bis 12. September 1964 stattgefundene Flottenübung der Volksmarine, 15. September 1964, SAPMO-BArch, DY 30/IV A2/12/65, o. Pag.

188 Vgl. Kap. III.3. dieser Studie. 
der weiteren Einführung und dem Einsatz neuer Kampftechnik im Verlaufe des Ausbildungsjahres ... wurde ihr Kampfwert und ihre Schlagkraft wesentlich erhöht " ${ }^{189}$ Offenbar war es gelungen, nicht zuletzt bei der Optimierung der militärischen Führungsprozesse und -strukturen gewisse Fortschritte zu erreichen. Dieses Ziel hatte vor allem im Zusammenhang mit der Integration der NVA in die Erste Strategische Staffel des Warschauer Paktes besondere Bedeutung. Hierzu meinte das Ministerium für Nationale Verteidigung, die „seit Anfang 1963 unternommenen Anstrengungen, die Truppenführung durch rationellere Führungsmethoden und Organisationsformen der Stabsarbeit sowie geeignete Maßnahmen der Mechanisierung und Automatisierung systematisch zu einer neuen Qualität zu entwickeln“, hätten „zu ersten sichtbaren Erfolgen“" geführt ${ }^{190}$.

Vor allem das Manöver „Oktobersturm“ im Oktober 1965 bewies, daß die NVA innerhalb von nur vier Jahren weitgehend das Leistungsniveau der übrigen Armeen der Vereinten Streitkräfte erreicht hatte und problemlos an integrierten Operationen der Ersten Strategischen Staffel des Warschauer Paktes teilnehmen konnte ${ }^{191}$. Im Hinblick auf den nunmehr erreichten Stand der Einsatzbereitschaft der DDRStreitkräfte formulierte der Chef der Politischen Hauptverwaltung Verner auf der 11. ZK-Tagung im Dezember 1965 daher als Fazit dieses Manövers: „Unter kompliziertesten Bedingungen haben unsere Soldaten, Unteroffiziere und Offiziere großartige und bisher nie dagewesene Leistungen vollbracht." 192

189 Beitrag zum Referat des Ministers: Auswertung des Ausbildungsjahres 1964/1965, BA-MA, VA-01/5181, Bl. 50.

190 Ebenda, Bl. 64.

191 Vgl. Kap. VII.1. dieser Studie.

192 Stenografisches Protokoll der 11. Tagung des ZK der SED, 15.-17. Dezember 1965, SAPMO-BArch, DY 30/IV 2/1/336, Bl. 165. 